\title{
Radiation From Electrons in Magnetoplasma
}

\author{
Harold B. Liemohn \\ Southwest Center for Advanced Studies, P.O. Box 30365, Dallas, Tex.
}

(Received August 4, 1964)

\begin{abstract}
The radiation from an electron in a homogeneous magnetoplasma has some unusual properties as a consequence of the dispersive anisotropic nature of the medium. Attention is confined to emission in the ordinary (whistler) mode frequency band below the cyclotron resonance and the extraordinary mode frequency band around the plasma frequency where the indices of refraction are appreciably greater than one and vary significantly. Due to the large indices, electrons can emit Cerenkov radiation over a limited band of nonrelativistic energies. The cyclotron radiation which is generated by the gyrations of electrons is complicated also by this property of the medium which permits both normal emission due to "slower than light" motion and anomalous emission due to "faster than light" motion. In the ordinary mode, for example, the anomalous cyclotron radiation is emitted into the forward hemisphere with respect to the guiding center motion of the electron whereas the normal radiation is emitted into the backward hemisphere. In this paper the frequency spectra and angular patterns of the average radiated power are calculated by the Hamiltonian method which avoids a direct calculation of the complicated electromagnetic field vectors. The theory of emission in dispersive anisotropic media with a hermitian dielectric tensor by Kolomenskii and Eidman is thoroughly reviewed and extended to include relativistic energies; the complicated analytic formulas for the power are evaluated for several special cases; and the results are applied to recent interpretations of very low-frequency (VLF) and low-frequency (LF) emissions from electrons in the magnetosphere. The main conclusions of the work are as follows: (1) In the ordinary (whistler) mode most of the energy is radiated along wave normals at large angles to the magnetic field at frequencies other than the rectilinearly Doppler-shifted fundamental cyclotron harmonic which is contrary to assumptions of certain VLF emission theories. (2) The resonance singularities in the indices for a cold, collisionless plasma must be eliminated to achieve finite power levels, but unfortunately the dielectric tensor for thermal motion is extremely complex and for collisions is non-hermitian; consequently, an arbitrary upper limit is imposed on the indices in order to make a quantitative estimate of the power. (3) Based on this approximation the total power in the ordinary mode is a slowly varying function of frequency and electron energy with an average level of $10^{-30} \mathrm{~W} /(\mathrm{c} / \mathrm{s})$ per electron. (4) This level is inadequate to explain observed VLF signals on the basis of incoherent emission, but coherent emission from bunches of electrons can give the observed power level of $10^{-14} \mathrm{~W} / \mathrm{cm}^{2}(\mathrm{c} / \mathrm{s})$ above the ionosphere; hence, the onus of explaining the complex dispersion patterns of VLF emissions is left to the coherence mechanism. (5) The radiation in the extraordinary mode varies considerably with frequency and energy but an average power level is on the order of $10^{-25} \mathrm{~W} /(\mathrm{c} / \mathrm{s})$ per electron which still requires some coherence to generate the observed level above the ionosphere; however, this energy cannot penetrate the ionosphere to account for the dispersion observed by ground-based receivers.
\end{abstract}

\section{Introduction}

The problem of radiation from an electron in a plasma in a homogeneous magnetic field occurs in astrophysics, radioastronomy, thermonuclear physics, and magnetospheric physics. Due to the dispersion and anisotropy of the magnetoplasma, the emission from an electron moving along a helical trajectory has some very unusual properties. The analysis is restricted to those frequency bands in the ordinary and extraordinary modes where the indices are appreciably greater than one. The polarization induced in the medium by the electronic charge produces Cerenkov radiation when the local phase velocity is less than the particle velocity, and acceleration due to the orbit gyrations of the charge produces cyclotron radiation which is appreciable in the low-order harmonics at Doppler-shifted frequencies. The cyclotron radiation consists of a "normal" emission in which the source moves slower than the local phase velocity and an "anomalous" emission in which the source moves faster.

The general nonrelativistic formulas for the frequency spectra and polar diagrams of the power radiated by gyrating electrons in a magnetoplasma have been given by Eidman [1958]. His analysis is based on earlier work by Kolomenskii [1953] in which the fundamental Hamiltonian method of

${ }^{1}$ This research has been supported by the National Aeronautics and Space Administration under grant NSG-269-62. 
calculating radiation from a source is generalized for the case of an anisotropic dispersive medium with a Hermitian dielectric tensor. Although the formulas are available, the emission properties have not been analyzed appreciably because extensive numerical evaluation is required. P. S. Johnson [1962] used the expressions to evaluate the frequency spectrum of Cerenkov radiation from an electron moving parallel to the magnetic field. The equation relating the frequency and angle of emission has been solved numerically by Ellis [1962] for certain parameters appropriate for radiation from Jupiter. The power formulas were also evaluated by Bazhanova and Shafranov [1963] for the special case of normal emission in the ordinary mode from the fundamental cyclotron harmonic. However, the general problem of Cerenkov radiation and normal and anomalous cyclotron radiation from several harmonics has not been considered for electrons in helical orbits.

Some approximate formulas have been derived independently. Several limiting cases of emission in a thermal magnetoplasma have been given by Pakhomov, Stepanov, and Aleksin [1962], and Pakhomov and Stepanov [1963a, 1963b]. Another method was employed recently by McKenzie [1963] to obtain the frequency spectra and polar diagram for Cerenkov radiation from a uniformly moving point charge.

This paper has three objectives. First, the complete theory by Eidman [1958] and Kolomenskii [1953] is reviewed in order to include relativistic effects and pick up certain errors and omissions in the original analysis. Second, a numerical study of the formulas for Cerenkov and cyclotron radiation is presented to demonstrate the unusual properties of the emissions. Third, the results are applied to recent interpretations of the very low-frequency (VLF) and low-frequency (LF) emissions from the magnetosphere (3 to $300 \mathrm{kc} / \mathrm{s})$.

As a first approximation, the medium is represented by a cold, collisionless electron plasma. However, at the resonance frequency where the index of refraction becomes infinite, the power is also infinite. By introducing additional physical effects such as thermal motion or collisions, the singularity is removed but the expressions become so complicated that numerical evaluation is very difficult. As a compromise, an upper bound is imposed on the index which cuts off the emission at a finite level. Fortunately, in many cases this cutoff model is a good approximation to the index when thermal and collisional effects are small, and the physical properties of the power are described quantitatively in some parameter regions.

The emission at high frequencies where the propagation is like that in a vacuum is not discussed in this paper. Attention is confined to frequencies near the cyclotron and plasma frequencies of the electrons where the indices are most interesting. In the ordinary (whistler) mode the emission is confined to frequencies below the cyclotron frequency. In the extraordinary mode the emission is confined to a narrow band of frequencies near the plasma frequency. The index of refraction for these modes is generally greater than unity, and the electric vector of the wave rotates in the same sense as the electrons gyrate about the field (right-hand polarization).

Due to the symmetry of the medium, the energy is radiated along cones about the magnetic field direction. The Cerenkov radiation is emitted entirely in the forward hemisphere with respect to the guiding center motion of the electron (velocity component along the static magnetic field). The normal cyclotron radiation is emitted entirely into the backward hemisphere in the ordinary mode, but into either or both hemispheres in the extraordinary mode. The anomalous cyclotron radiation is emitted entirely into the forward hemisphere. In general, the maximum power is radiated at an appreciable angle to the axis of symmetry.

The anomalous cyclotron radiation is a consequence of the anomalous Doppler effect which was discussed some time ago by Franck [1942]. Since this property only arises when the source moves faster than the local phase velocity of the medium, the physical process is analogous to the Cerenkov mechanism; the charge induces a polarized wake in the medium which radiates electromagnetic energy. An elementary example of the anomalous effect occurs in the acoustic case where the source moves faster than the speed of sound, and a stationary observer receives both increased and decreased (Doppler-shifted) frequencies when the source is receding but no signal when the source is approaching. 
Finally, the feasibility of Cerenkov or cyclotron radiation from electrons as the source of VLF and LF emissions in the magnetosphere is discussed. A review of the early observations and theories and a classification system for the various emissions has been prepared by Gallet [1959]. The frequency-time patterns range from continuous wide bands of noise called hiss to discrete patterns with descriptive class titles: hooks, risers, quasi-vertical ("dawn chorus"), etc. The intensity of the wide band noise has been measured simultaneously at several frequencies by Dowden [1962a], and after ionospheric absorption effects were removed it was concluded that the source spectrum is relatively flat at a level of the order of $10^{-14} \mathrm{~W} / \mathrm{cm}^{2}(\mathrm{c} / \mathrm{s})$. The morphology of VLF emission events has been recorded by Watts, Koch, and Gallet [1963] using an instrument which permits continuous observations. Recently, Helliwell [1963] has found that certain periodic VLF emissions are triggered by whistler echoes.

In addition to these ground-based observations, VLF emissions have been observed above the ionosphere by satellites. The VLF receiver aboard the Alouette satellite was operated by Barrington and Belrose [1963]. Their telemetered recordings of wide band noise exhibit a systematic decrease in the lower cutoff frequency as the satellite latitude increases. More detailed VLF emission data was obtained by Gurnett [1963] with instrumentation aboard the Injun III satellite. In addition to the telemetered wide band signal, a six channel frequency spectrum analyzer measured the absolute amplitude of the VLF magnetic component. The rms amplitude at the satellite varied from the threshold of $10^{-3}$ to a maximum of $5 \times 10^{-2}$ gamma $\left(\sim 10^{-14} \mathrm{~W} / \mathrm{cm}^{2}(\mathrm{c} / \mathrm{s})\right)$. On several occasions complex VLF emissions were triggered by spheric impulses. A clear correlation among VLF emissions, auroral enhancements, and electron precipitation was also revealed. In a rocket flight to $1700 \mathrm{~km}$, Walsh, Haddock, and Schulte [1963] observed intense radio noise near $1 \mathrm{Mc} / \mathrm{s}$, which they attribute to emissions in both modes.

Several mechanisms have been suggested as the source of VLF emissions. Gallet and Helliwell [1959] proposed that geomagnetic field alined streams of electrons interact, as in the traveling wave tube mechanism, to produce amplification of existing background electromagnetic radiation. This mechanism was recently considered in greater detail by Dowden [1962b] who showed that traveling wave tube amplification of selected bands of VLF noise can explain several characteristics of hiss. The possibility of Doppler-shifted VLF cyclotron radiation from protons was proposed by MacArthur [1959] and later extended by Murcray and Pope [1960a, 1960b]. Dowden [1962c, 1963] also showed that Doppler-shifted cyclotron emissions from discrete bunches of electrons produce hook dispersion patterns which fit the experimental data quite well. Bell and Buneman [1964] found a plasma instability in the interaction between a whistler-mode wave and a gyrating-electron stream which may initiate coherent triggered emissions. Triggering mechanisms and cyclotron emissions are also discussed by Hansen [1963]. The transfer of energy between whistler-mode signals and energetic charged particles has been examined for several VLF emission mechanisms by Brice [1964].

In most of these theoretical analyses only the one-dimensional longitudinal equations (parallel to the geomagnetic field) were considered so that wave normal directions in which the maximum Cerenkov and cyclotron power is available were tacitly omitted. Furthermore, no estimates of the total power or its spectrum were attempted. With the power estimates presented here it is demonstrated that incoherent emission is undoubtedly inadequate, but coherent emission from bunches of electrons may provide the observed power level.

\section{Hamiltonian Method}

The Hamiltonian method for calculating the energy radiated by a charged particle in an anisotropic dispersive medium is described in some detail. The solution which was formulated by Kolomenskii [1953] is a generalization of a method employed by Heitler [1954 and earlier editions] and others for calculating particle emissions in free space. The name of the method arises because the coefficients in the eigenwave expansion for the vector potential of the radiation field are just the canonical variables of the Hamiltonian for the system. 
Any homogeneous medium which is electromagnetically dispersive and anisotropic is characterized by a complex dielectric tensor $\underline{\underline{\epsilon}}(\omega, \theta, \phi)$, where $\omega$ is the angular propagation frequency and $\theta$ and $\phi$ are the polar and azimuthal angles measured from some reference direction. It is necessary for the following analysis to assume that this tensor is Hermitian

$$
\underline{\underline{\epsilon}}=\underline{\underline{\epsilon}}^{*}
$$

where the asterisk denotes the complex conjugate and transpose. Furthermore, the magnetic susceptibility of the medium is assumed to vanish so that the permeability $\mu$ of the medium is that of free space. The implications of these assumptions in a magneto-ionic medium are discussed in the next section.

A charged particle moving through such a medium may radiate electromagnetic energy if it is being accelerated or if its velocity is greater than the phase velocity of the medium. The electric and magnetic fields which are generated by a particle with charge $e$, trajectory $\mathbf{r}_{e}$ and velocity $\mathbf{v}$ are described by Maxwell's field equations (Gaussian units) and some familiar subsidiary relations:

$$
\begin{gathered}
\nabla \times \boldsymbol{H}-\dot{\boldsymbol{D}} / c=4 \pi(e / c) \mathbf{v} \delta\left(\mathbf{r}-\mathbf{r}_{e}\right) \\
\nabla \times \boldsymbol{E}+\dot{\boldsymbol{B}} / c=0 \\
\nabla \cdot \boldsymbol{B}=0 \\
\nabla \cdot \boldsymbol{D}=4 \pi e \delta\left(\mathbf{r}-\mathbf{r}_{e}\right) \\
\boldsymbol{D}=\boldsymbol{\epsilon} \cdot \boldsymbol{E}, \boldsymbol{B}=\boldsymbol{H}
\end{gathered}
$$

where $\delta$ is the Dirac delta function and the dot denotes $\partial / \partial t$.

By introducing the electromagnetic potentials, $\mathbf{A}$ and $\Phi$, which are defined by the field quantitites,

$$
\begin{gathered}
\boldsymbol{E}=-\nabla \Phi-\mathbf{A} / c, \\
\boldsymbol{B}=\nabla \times \mathbf{A},
\end{gathered}
$$

equations (2.2) to (2.6) reduce to

$$
\begin{gathered}
\nabla \times(\nabla \times \mathbf{A})+\underline{\underline{\epsilon}} \cdot \nabla \Phi / c+\underline{\underline{\epsilon}} \cdot \ddot{\mathbf{A}} / c^{2}=4 \pi(e / c) \mathbf{v} \delta\left(\mathbf{r}-\mathbf{r}_{e}\right), \\
-\nabla \cdot \underline{\underline{\epsilon}} \cdot \nabla \Phi-\nabla \cdot \underline{\underline{\epsilon}} \cdot \dot{\mathbf{A}} / c=4 \pi e \delta\left(\mathbf{r}-\mathbf{r}_{e}\right) .
\end{gathered}
$$

Since the potentials are not uniquely defined by (2.7) and (2.8) a Coulomb gage condition may be imposed on them:

$$
\nabla \cdot \underline{\underline{\epsilon}} \cdot \mathbf{A}=0
$$

In this gage, $\Phi$ is just the static potential of the source charge and $\boldsymbol{D}_{\mathrm{st}}=\underline{\underline{\epsilon}} \cdot \nabla \Phi$ is the corresponding static vector field. It will be shown that the electromagnetic radiatiòn fields $\boldsymbol{E}_{\mathrm{em}}$ and $\boldsymbol{B}_{\mathrm{em}}$ are derivable from the vector potential A alone.

In order to express these equations in canonical form it is necessary to consider only that part of the field which lies inside an arbitrarily large but finite cubic volume $\tau=L^{3}$, where $L \gg$ charac- 
teristic wavelength. In this volume the potentials are represented by a superposition of eigenfunctions $A_{\lambda}$ and $\Phi_{\lambda}$,

$$
\begin{aligned}
& \mathbf{A}=\sum_{\lambda} q_{\lambda}(t) \mathbf{A}_{\lambda}(\mathbf{r}), \\
& \Phi=\sum_{\lambda} q_{0 \lambda}(t) \Phi_{\lambda}(\mathbf{r}),
\end{aligned}
$$

where $q_{\lambda}$ and $q_{0 \lambda}$ are expansion coefficients. The eigenfunctions are required to satisfy the boundary conditions,

$$
\mathbf{A}_{\lambda}, \nabla \times \mathbf{A}_{\lambda}, \Phi_{\lambda}, \nabla \Phi_{\lambda}
$$

periodic on the surface $\sigma$ of the volume $\tau$,

and the homogeneous wave equations,

$$
\begin{aligned}
& \nabla \times\left(\nabla \times \mathbf{A}_{\lambda}\right)-\omega_{\lambda}{ }^{2} \underline{\underline{\epsilon}} \cdot \mathbf{A}_{\lambda} / c^{2}=0, \\
& \nabla^{2}\left(\nabla \Phi_{\lambda}\right)+\omega_{0}{ }_{\lambda} \underset{\underline{\epsilon}}{=} \cdot \nabla \Phi_{\lambda} / c^{2}=0,
\end{aligned}
$$

where the eigenvalues $\omega_{\lambda}$ and $\omega_{0 \lambda}$ are real constants. Since the divergence of a curl always vanishes, the eigenfunctions $\mathbf{A}_{\lambda}$ defined by (2.14) also satisfy (2.11) as required. The orthogonality of the set of $\mathbf{A}_{\lambda}$ with respect to the weighting function $\epsilon$ can be verified using (2.1), (2.13), and (2.14) and the divergence theorem,

$$
\begin{aligned}
\left(\omega_{\mu}^{2}-\omega_{\lambda}^{2}\right) \int d \tau A_{\mu}^{*} \cdot \underline{\underline{\epsilon}} \cdot A_{\lambda} & =c^{2} \int d \tau\left[\mathbf{A}_{\lambda} \cdot \nabla \times \nabla \times \mathbf{A}_{\mu}^{*}-\mathbf{A}_{\mu}^{*} \cdot \nabla \times \nabla \times \mathbf{A}_{\lambda}\right] \\
& =c^{2} \int d \tau \nabla \cdot\left[\mathbf{A}_{\mu}^{*} \times \nabla \times \mathbf{A}_{\lambda}-\mathbf{A}_{\lambda}^{*} \times \nabla \times \mathbf{A}_{\mu}\right]=0, \quad(\lambda \neq \mu) .
\end{aligned}
$$

The orthogonality of the set of $\nabla \Phi_{\lambda}$ with respect to $\epsilon$ is verified analogously. The normalization of the eigenfunctions is chosen such that

$$
\begin{aligned}
& \int d \tau \mathbf{A}_{\mu}^{*} \cdot \underline{\underline{\epsilon}} \cdot \mathbf{A}_{\lambda}=4 \pi c^{2} \delta_{\lambda \mu}, \\
& \int d \tau \nabla \Phi_{\mu}^{*} \cdot \epsilon \cdot \nabla \Phi_{\lambda}=4 \pi c^{2} \delta_{\lambda \mu} .
\end{aligned}
$$

The orthogonality of $\mathbf{A}_{\lambda}$ and $\nabla \Phi_{\mu}$ for all $\lambda, \mu$ with respect to the weighting function $\underline{\underline{\epsilon}}$ can be proved with the aid of a vector identity,

$$
\int d \tau[\mathbf{b} \cdot \nabla \times(\nabla \times \mathbf{a})-\nabla \times \mathbf{b} \cdot \nabla \times \mathbf{a}]=-\int d \boldsymbol{\sigma} \cdot(\mathbf{b} \times \nabla \times \mathbf{a}) .
$$

Inserting $\mathbf{a}=\mathbf{A}_{\lambda}$ and $\mathbf{b}=\nabla \Phi_{\mu}^{*}$, the surface integral vanishes due to the boundary conditions and the last term of the volume integral obviously vanishes to that with the aid of (2.1) and (2.14)

$$
\int d \tau \mathbf{A}_{\lambda}^{*} \cdot \underline{\underline{\epsilon}} \cdot \nabla \Phi_{\mu}=\left[\int d \tau \nabla \Phi_{\mu}^{*} \cdot \epsilon \cdot \mathbf{A}_{\lambda}\right]^{*}=0 .
$$

The expansions for $\mathbf{A}$ and $\Phi$ permit (2.9) and (2.10) to be reduced to a system of uncoupled elementary scalar equations for $q_{\lambda}$ and $q_{0 \lambda}$. If (2.9) is multiplied by $\mathbf{A}_{\mu}^{*}$ and integrated over $\tau$, the orthogonality relations derived above reduce the vector equation to simple harmonic oscillator 
equations for the set of $q_{\mu}$,

$$
\ddot{q}_{\mu}+\omega_{\mu}^{2} q_{\mu}=(e / c) \mathbf{v} \cdot \mathbf{A}_{\mu}^{*}\left(\mathbf{r}_{e}\right)
$$

The forcing function depends on the dielectric tensor since $\mathbf{A}_{\mu}^{*}$ must satisfy (2.14), and on the time through $\mathbf{r}_{e}$ and $\mathbf{v}$. Similarly multiplying (2.9) and (2.10) by $\nabla \Phi_{\mu}^{*}$ and integrating over $\tau$ yields

$$
\begin{aligned}
c \dot{q}_{0 \mu} & =(e / c) \mathbf{v} \cdot \nabla \Phi_{\mu}^{*}\left(\mathbf{r}_{e}\right), \\
c^{2} q_{0 \mu} & =e \Phi_{\mu}^{*}\left(\mathbf{r}_{e}\right),
\end{aligned}
$$

respectively. Since $\mathbf{r}_{e}$ is explicitly time dependent, $\partial \Phi_{\mu}^{*}\left(\mathbf{r}_{e}\right) / \partial t=\mathbf{v} \cdot \nabla \Phi_{\mu}^{*}\left(\mathbf{r}_{e}\right)$, and therefore $\left(2.21^{\prime}\right)$ is redundant because it is just the time derivative of (2.21). Hence, the complete solution for the static and electromagnetic fields can be obtained in principle by solving (2.20) and (2.21) with the appropriate set of $\mathbf{A}_{\mu}$ and $\Phi_{\mu}$ for the medium.

Since $A$ and $\Phi$ are determined by uncoupled systems of equations, the electric and magnetic fields which they prescribe are also uncoupled. In particular, the electromagnetic fields are obtained from the vector potential alone using (2.7) and (2.8),

$$
\boldsymbol{E}_{e m}=-\dot{\mathbf{A}} / c, \quad \boldsymbol{B}_{e m}=\nabla \times \mathbf{A} .
$$

In the present application only the energy of the radiation field is required, and it is given by the general expression

$$
W=\frac{1}{4 \pi} \int d t^{\prime} \int d \tau\left(\boldsymbol{E}_{e m}^{*} \cdot \boldsymbol{D}_{e m}+\dot{\boldsymbol{B}}_{e m}^{*} \cdot \boldsymbol{H}_{e m}\right)
$$

The magnetic energy term is simplified with the aid of (2.18). Inserting $\mathbf{a}=\mathbf{A}_{\lambda}$ and $\mathbf{b}=\mathbf{A}_{\mu}^{*}$, noting that the surface integral vanishes by the boundary conditions, and using (2.14), it is found that

$$
\int d \tau \nabla \times \mathbf{A}_{\mu}^{*} \cdot \nabla \times A_{\lambda}=\left(\omega_{\lambda}^{2} / c^{2}\right) \int d \tau \mathbf{A}_{\mu}^{*} \cdot \underline{\underline{\epsilon}} \cdot \mathbf{A}_{\lambda} .
$$

Hence, using the normalization of (2.17), (2.23) reduces to

$$
W(t)=\int d t^{\prime} \sum_{\mu}\left(\dot{q}^{*} \ddot{q}+\omega_{\mu}^{2} \dot{q}_{\mu}^{*} q_{\mu}\right)=\frac{1}{2} \sum_{\mu}\left(\dot{q}_{\mu}^{*} \dot{q}_{\mu}+\omega_{\mu}^{2} q_{\mu}^{*} q_{\mu}\right)=\sum_{\mu} w_{\mu}
$$

This expression is identical to that for the Hamiltonian of a system of harmonic oscillators which describe the radiation field.

The polarization of the electromagnetic waves can take two arbitrary directions which correspond to the two possible modes of propagation in the medium. In the foregoing equations these different modes of polarization are denoted by different indices, $\lambda($ or $\mu)$. In the following equations only one mode is considered.

In order to evaluate the summation in (2.25) it is necessary to assume a particular form for the eigenvectors $\mathbf{A}_{\mu}$ which satisfy (2.13), (2.14), and (2.17). Here, for convenience, they are chosen to be

$$
\mathbf{A}_{\mu}=\sqrt{4 \pi} c\left(\mathbf{a}_{\mu} / n_{\mu} L^{3 / 2}\right) \exp \left(-i \mathbf{k}_{\mu} \cdot \mathbf{r}\right)
$$

where $\mathbf{a}_{\mu}$ is the polarization vector, $n_{\mu}$ is the index of refraction, and $\mathbf{k}_{\mu}$ is the propagation vector. 
(The sign convention in the exponent is opposite to that used by Kolomenskii and Eidman, but it is essential for the equations to be consistent with the polarization convention used in magnetoionic theory.) The orthogonality of $\mathbf{A}_{\mu}$ in $\tau$ is assured if the components of $\mathbf{k}_{\mu}$ are given by $k_{\mu} \eta$ $=2 \pi \mu_{\eta} / L$ where the $\mu_{\eta}$ are integers and $\eta=x, y, z$. The components of $\mathbf{a}_{\mu}$ and $\mathrm{n}_{\mu}$ are determined, in principle, by (2.14) and (2.17) which reduce to

$$
\left(c^{2} / \omega_{\mu}^{2}\right)\left[k_{\mu}^{2} \mathbf{a}_{\mu}-\mathbf{k}_{\mu}\left(\mathbf{k}_{\mu} \cdot \mathbf{a}_{\mu}\right)\right]=\underline{\underline{\epsilon}} \cdot \mathbf{a}_{\mu}, \quad \mathbf{a}_{\mu}^{*} \cdot \underline{\underline{\epsilon}} \cdot \mathbf{a}_{\mu}=n_{\mu}^{2} .
$$

Since the wavelength is small compared to $L(L \rightarrow \infty), k$-space is approximately continuous and $\mu$-space can be represented by continuous spherical coordinates $\mu, \theta, \phi$ without loss of generality. (The polar and azimuthal angles, $\theta$ and $\phi$, are independent of the space variables in $\tau$.) Furthermore, by definition, $\omega n / c=k=2 \pi \mu / L$ so that the frequency $\omega$ will be used as the independent variable with $k(\omega, \theta, \phi), n(\omega, \theta, \phi)$, and $\mathbf{a}(\omega, \theta, \phi)$, in general, and the subscript $\mu$ is omitted hereafter.

The number of oscillators in $\tau$ which radiate into the solid angle $d \Omega=\sin \theta d \theta d \phi$ and the frequency interval between $\omega$ and $\omega+d \omega$ for a prescribed polarization is given by

$$
\tau d^{3} \mu=\mu^{2} d \mu d \Omega \tau=\frac{\omega^{2} n^{3} L^{3}}{(2 \pi c)^{3}}\left(1+\frac{\omega}{n} \frac{\partial n}{\partial \omega}\right) d \omega d \Omega \tau=\rho d \omega d \Omega \tau,
$$

where $\rho(\omega, \theta, \phi)$ is the density function of the radiation. (In Kolomenskii's [1953] and Eidman's [1958] papers the frequency dependence of $n$ was omitted in the definition of $\rho$.)

With a continuous variable for $\mu$ the discrete summation in (2.25) transforms into a volume integral over the continuous variable $w$. Hence, the differential energy of the radiation field can be expressed as

$$
d^{3} W=w \rho d \omega d \Omega
$$

where $w(\omega, \theta, \phi, t)$ is the energy of a forced oxcillator in $d \omega d \Omega$.

\section{Emission in a Magneto-Ionic Medium}

The foregoing general equations are developed further in this section for emission in a magnetoionic medium which consists of a uniform static magnetic field and a homogeneous electrically neutral plasma. Such a medium is dispersive and anisotropic with respect to the direction of the magnetic field. The analysis follows Eidman's method with some modifications and corrections.

The $x y z$ coordinate system is oriented such that the $z$-axis is parallel to the static field $\mathbf{B}_{0}$. If collisions are neglected, the orbit of a relativistic electron in the static field consists of a gyration about the field direction and a drift along it as shown in figure 1 . Hence, the position $\left(\mathbf{r}_{e}\right)$ and

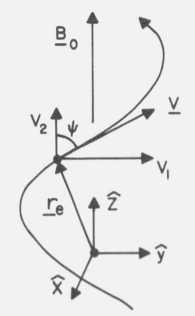

FigURE 1. The position and velocity coordinates of an electron on a helical trajectory. 


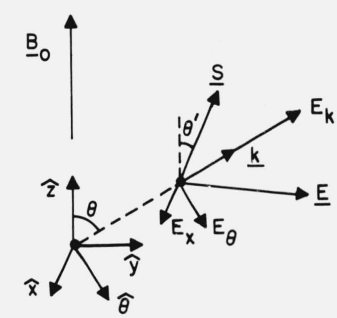

FIGURE 2. The components of the electric vector $\mathbf{E}$ of the radiation field in a magnetoplasma.

The wave normal $\mathbf{k}$ and the Poynting vector $\mathbf{S}$ are not parallel in general.

velocity $(\mathbf{v})$ coordinates along the $x, y, z$ axes are, respectively,

$$
\begin{array}{ccc}
\gamma r_{1} \cos \gamma^{-1} \omega_{B} t, & \gamma r_{1} \sin \gamma^{-1} \omega_{B} t, & v_{2} t, \\
-v_{1} \sin \gamma^{-1} \omega_{B} t, & v_{1} \cos \gamma^{-1} \omega_{B} t, & v_{2},
\end{array}
$$

where the subscripts 1 and 2 refer to components perpendicular and parallel to $\mathbf{B}_{0}$ and the relativistic corrections have been introduced explicitly. The nonrelativistic cyclotron or gyrofrequency is related to $\mathbf{B}_{0}$ by $\omega_{B}=|e| \mathbf{B}_{0} / \mathrm{Mc}$, and the nonrelativistic gyroradius is given by $r_{1}=v_{1} / \omega_{B}$. The relativistic factor $\gamma$ is defined as usual by

$$
\gamma=\left(1-\beta^{2}\right)^{-1 / 2}, \quad \beta^{2}=\left(v_{1}^{2}+v_{2}^{2}\right) / c^{2} .
$$

The propagation vector $\mathbf{k}$ is at some angle $\theta$ with respect to $\mathbf{B}_{0}$, in general, and it is arbitrarily placed in the $y z$ plane since there is azimuthal symmetry,

$$
\mathbf{k}=k \hat{k}=k \sin \theta \hat{y}+k \cos \theta \hat{z}
$$

where $\hat{k}, \hat{y}, \hat{z}$ are unit vectors. The components of the electromagnetic fields are taken along the orthogonal directions $\hat{x}, \hat{\theta}, \hat{k}$ as shown in figure 2 .

The dielectric tensor for a magneto-ionic medium can have several forms depending on which properties of the plasma are important. In addition to the frequency domain, the effect of thermal motion, collisions, or ionic motion alter the dielectric tensor appreciably under certain conditions. Elementary derivations of the tensor for a cold plasma are given by Ratcliffe [1959], Ginzburg [1961], and others. Several papers have treated electromagnetic wave propagation in hot plasmas recently, and an extensive review is provided by Stix [1962]. It is noteworthy that the tensor is hermitian when thermal motion or ionic motion is included, but is not hermitian when collisions are important.

The polarization of the electric vector of the wave field and the index of refraction for the medium are obtained from the components of the dielectric tensor. In terms of the spatial configuration in figure 2 the polarization coefficients are defined by

$$
E_{\theta} / E_{x}=i \alpha_{\theta}(\omega, \theta), \quad E_{k} / E_{x}=i \alpha_{k}(\omega, \theta),
$$

and the index is denoted by $n(\omega, \theta)$, where $\alpha_{\theta}$ and $\alpha_{k}$ may be complex but $n$ must be real in the following solution.

The components of $\mathbf{a}$ (and $n$ ) in this medium are determined by (2.27) and (2.28) for a prescribed $\underline{\underline{\epsilon}}$. Since the electric field of the propagating wave must satisfy the wave equation, its amplitude 
vector $\mathbf{E}$ must satisfy (2.27). Hence, the solution of these four complicated algebraic equations is obtained indirectly by assuming that $\mathbf{a}=\kappa \mathbf{E} / E_{x}$. The constant of proportionality is easily shown to be

$$
\kappa=\left(1+\left|E_{\theta} / E_{x}\right|^{2}\right)^{-1 / 2}=\left(1+\alpha_{\theta}^{2}\right)^{-1 / 2}
$$

(Eidman's normalization factor $\kappa=1 / \sqrt{2}$ does not satisfy the equations unless $\cos \theta= \pm 1$.) Therefore, the components of $\mathbf{a}$ in the Cartesian coordinate system of the electron are given by

$$
a_{x}=\kappa, \quad a_{y}=i \kappa \alpha_{y}, \quad a_{z}=i \kappa \alpha_{z},
$$

where

$$
\begin{aligned}
& \alpha_{y}=\alpha_{\theta} \cos \theta+\alpha_{k} \sin \theta, \\
& \alpha_{z}=\alpha_{k} \cos \theta-\alpha_{\theta} \sin \theta .
\end{aligned}
$$

With the foregoing explicit expressions for $\mathbf{A}, \mathbf{r}_{e}, \mathbf{v}, \mathbf{k}$, and a given by (2.26), (3.1), (3.2), (3.4), and (3.7), respectively, the forcing function in (2.20) takes the form

$$
\begin{aligned}
(e / c) \mathbf{v} \cdot \mathbf{A}^{*}=-\frac{\sqrt{4 \pi} i e \kappa}{n L^{3 / 2}}\left(-i v_{1} \sin \gamma^{-1} \omega_{B} t\right. & \left.+\alpha_{y} v_{1} \cos \gamma^{-1} \omega_{B} t+\alpha_{z} v_{2}\right) \\
& \exp \left(+i k \sin \theta \quad \gamma r_{1} \sin \gamma^{-1} \omega_{B} t+i k \cos \theta \quad v_{2} t\right) .
\end{aligned}
$$

This expression can be simplified by introducing the Bessel function expansion

$$
\exp (+i X \sin \phi)=\sum_{-\infty}^{+\infty} J_{s}(X) \exp (+i s \phi)
$$

so that

$\left(\alpha_{y} \cos \phi-i \sin \phi\right) \exp (+i X \sin \phi)$

$$
=\frac{1}{2} \sum_{-\infty}^{+\infty}\left(\alpha_{y} J_{s+1}+\alpha_{y} J_{s-1}+J_{s+1}-J_{s-1}\right) \exp (+i s \phi)=\sum_{-\infty}^{+\infty}\left(-J_{s}^{\prime}+\alpha_{y} s J_{s} / X\right) \exp (+i s \phi)
$$

where the prime denotes differentiation with respect to $X$. Hence, (2.20) for the forced harmonic oscillator has the simple form

$$
\ddot{q}+\omega^{2} q=\sum_{-\infty}^{+\infty} C_{s} \exp \left(+i p_{s} t\right)
$$

where

$$
p_{s}(\omega, \theta)=s \omega_{B} / \gamma+v_{2} k \cos \theta=s \omega_{B} / \gamma+\beta_{2} \omega n(\omega, \theta) \cos \theta
$$

and

$$
C_{s}(\omega, \theta)=-\frac{\sqrt{4 \pi} i e c \kappa}{n L^{3 / 2}}\left[-\beta_{1} J_{s}^{\prime}(X)+\left(\alpha_{y} s \beta_{1} / X+\alpha_{z} \beta_{2}\right) J_{s}(X)\right],
$$


with

$$
X(\omega, \theta)=\gamma r_{1} k \sin \theta=\gamma \beta_{1} n(\omega, \theta)\left(\omega / \omega_{B}\right) \sin \theta
$$

and

$$
\beta_{1}=v_{1} / c, \quad \beta_{2}=v_{2} / c
$$

With the initial conditions $q=\dot{q}=0$ at $t=0$, the general solution of (3.12) is

$$
q=\sum_{-\infty}^{+\infty} q_{s}=\sum_{-\infty}^{+\infty} C_{s}\left[\frac{\exp \left(i p_{s} t\right)-\exp (+i \omega t)}{2 \omega\left(\omega-p_{s}\right)}+\frac{\exp \left(i p_{s} t\right)-\exp (-i \omega t)}{2 \omega\left(\omega+p_{s}\right)}\right] .
$$

The sum of the energies of all the modes then vanishes at $t=0$ and is given by

$$
\begin{aligned}
w & =\sum_{-\infty}^{+\infty} \frac{1}{2}\left(\dot{q}_{s}^{*} \dot{q}_{s}+\omega^{2} q_{s}^{*} q_{s}\right)+\text { cross product terms } \\
& =\sum_{-\infty}^{+\infty} \frac{1}{2}\left|C_{s}\right|^{2}\left[\frac{1-\cos \left(\omega-p_{s}\right) T}{\left(\omega-p_{s}\right)^{2}}+\frac{1-\cos \left(\omega+p_{s}\right) T}{\left(\omega+p_{s}\right)^{2}}\right]+\begin{array}{l}
\text { oscillating cross } \\
\text { product terms }
\end{array}
\end{aligned}
$$

at $t=T$. In the limit $T \rightarrow \infty$, the explicit summation terms reduce to Dirac delta functions,

$$
\operatorname{Lim}_{T \rightarrow \infty} \frac{1-\cos \left(\omega \pm p_{s}\right) T}{\pi\left(\omega \pm p_{s}\right)^{2} T}=\operatorname{Lim}_{T \rightarrow \infty} \frac{\sin \left(\omega \pm p_{s}\right) T}{\pi\left(\omega \pm p_{s}\right)}=\delta\left(\omega \pm p_{s}\right) .
$$

The first equality can be demonstrated by partial integration, and the second is discussed by Schiff [1955]. The average (unweighted differential) energy per unit time radiated into $\tau$ by the electron is given by

$$
\operatorname{Lim}_{T \rightarrow \infty} w / T=\frac{\pi}{2} \sum_{-\infty}^{+\infty}\left|C_{s}\right|^{2}\left[\delta\left(\omega-p_{s}\right)+\delta\left(\omega+p_{s}\right)\right]
$$

where the oscillating transient terms vanish as $1 / T$.

The limiting process which introduces the $\delta$-functions is justified provided that the radiation never reaches the boundary $\sigma$ of the volume $\tau$. This is assured because $L$ and $T$ can approach infinity and simultaneously satisfy the inequality $L \gg c T / n$.

The mathematical interpretation of the $\delta$-function terms depends on the propagation properties of the vector-potential quantity

$$
q_{\mu} \mathbf{A}_{\mu}=q_{\omega, \theta, \phi}(t) \mathbf{A}_{\omega, \theta, \phi}(\mathbf{r}) \sim \exp i( \pm \omega t-\mathbf{k} \cdot \mathbf{r}),
$$

where $q$ is given by (3.17), $\mathbf{A}$ is given by (2.26), and $\omega$ is always positive. If a particular set of values, $\omega_{1}, s_{1} \geqslant 0, \theta_{1}$, satisfy $\omega=+p_{s}$, then by (3.13), $\omega_{1},-s_{1}, \theta_{1}+\pi$ satisfy $\omega=-p_{s}$. Therefore, the term $\delta\left(\omega-p_{s}\right)$ which is associated with the first term in (3.17) has a propagation exponent of the form $+\omega_{1} t-k_{1} y \sin \theta_{1}-k_{1} z \cos \theta_{1}$, where $k=\omega n / c$, and the term $\delta\left(\omega+p_{s}\right)$ which is associated with the second term in (3.17) has a propagation exponent of the form $-\omega_{1} t+k_{1} y \sin \theta_{1}+k_{1} z$ $\cos \theta_{1}$. Hence, these two terms describe identical radiation which is called "normal" emission because of its dispersion relation. Similarly, if $\omega_{2}, s_{2}<0, \theta_{2}$ satisfy $\omega=+p_{s}$, then $\omega_{2},-s_{2}, \theta_{2}+\pi$ satisfy $\omega=-p_{s}$, and the two terms have a common propagation exponent of the form $\pm\left(\omega_{2} t-k_{2} y\right.$ $\sin \theta_{2}-k_{2} z \cos \theta_{2}$ ). This radiation is called "anomalous" because of its unconventional dispersion relation which is discussed below. 
Due to this duplication of emission properties, the energy expression can be simplified. By introducing the explicit property $\alpha_{\theta, k}(\omega, \theta)=-\alpha_{\theta, k}(\omega, \theta+\pi)$, which is valid in the magneto-ionic medium, and using the properties of Bessel functions it is readily verified that $C_{s}(\omega, \theta)$ $=-C_{-s}(\omega, \theta+\pi)$. Therefore, the energies given by $\delta\left(\omega-p_{s}\right)$ and $\delta\left(\omega+p_{s}\right)$ are identical, and the radiation emitted by the electron is described completely by the terms

$$
\operatorname{Lim}_{T \rightarrow \infty} w_{s} / T=\pi\left|C_{s}\right|^{2} \delta\left(\omega-p_{s}\right),
$$

where $s=0, \pm 1, \pm 2, \ldots$ The frequency $\omega$ and polar angle $\theta$ of the emission are related by the anisotropic dispersion equation $\omega=+p_{s}$, henceforth called the emission equation, which can be expressed in the form

$$
\beta_{2} \cos \theta_{s}=\left(\omega-s \omega_{B} / \gamma\right) / \omega n\left(\omega, \theta_{s}\right)
$$

The physical explanation of the emission terms is provided by the properties of the emission equation. The $s=0$ term describes Cerenkov radiation, emission from induced polarization in the medium, because $\theta_{0}$ must satisfy the usual relation $\cos \theta_{0}=1 / \beta_{2} n$. Since $n$ is independent of the sign on the static-field vector-component, the Cerenkov emission is in the forward hemisphere with respect to the velocity component $\beta_{2}\left(\beta_{2} \cos \theta_{0}>0\right)$. For $s \neq 0$ the emission is due to the cyclotron harmonics $|s| \omega_{B} / \gamma$. The distinction between the normal and anomalous emission is obtained from the emission equation relation $\beta_{2} n \cos \theta_{s} \lessgtr 1$ for $s \gtrless 0$. Hence, the guiding center moves slower than the parallel component of the phase velocity when $s>0$ and a normal Doppler effect is obtained, but it moves faster when $s<0$ and an anomalous effect is obtained. The normal emission appears in either the forward or backward hemisphere $\left(\beta_{2} \cos \theta_{s} \gtrless 0\right)$ depending on whether $\omega \gtrless s \omega_{B} / \gamma$, whereas all the anomalous emission is in the forward hemisphere $\left(\beta_{2} \cos \theta_{s}\right.$ $>0$ ). Hence, frequencies below the source frequency $|s| \omega_{B} / \gamma$ are emitted into both hemispheres when the propagation modes permit. The simultaneous emission of both the normal and anomalous radiation is due to the dispersive anisotropic nature of the medium which allows waves of a given frequency to be reinforced in many directions at once. The general solution of (3.23) for $\cos \theta_{s}(\omega)$ and its application to VLF and LF emissions in the magnetosphere is considered in the following section.

The Cerenkov and cyclotron power which is radiated by the electron into $d \omega d \Omega$ is given by terms of the form

$$
d^{3} P_{s}=\operatorname{Lim}_{T \rightarrow \infty}\left(w_{s} / T\right) \rho d \omega d \Omega, \quad s=0, \pm 1, \pm 2, \ldots
$$

where the definition of average power and (2.30) have been used. In order to perform the integration over frequency or solid angle, the delta function must be reduced to an elementary form by the expansion,

$$
\delta(f(x))=\delta\left[\left(x-x_{s}\right) f^{\prime}\left(x_{s}\right)\right]=\left|f^{\prime}\left(x_{s}\right)\right|^{-1} \delta\left(x-x_{s}\right),
$$

where $x=\theta$ or $\omega$ and $x_{s}$ is that value of $\theta$ or $\omega$ for which $\omega=p_{s}$. Therefore, with $\rho$ and $C_{s}$ given by (2.29) and (3.14), the power radiated into the linear frequency interval $d f=d \omega / 2 \pi$ is obtained by a trivial integration,

$d P_{s} / d f=\left(2 \pi e^{2} / c \beta_{2}\right) \omega\left\{\kappa^{2}\left[-\beta_{1} J_{s}^{\prime}(X)+\left(\alpha_{y} s \beta_{1} / X+\alpha_{z} \beta_{2}\right) J_{s}(X)\right]^{2}\right.$

$$
\cdot[1+(\omega / n) \partial n / \partial \omega] /|1-(\partial n / \partial \theta) / n \tan \theta|\}_{\theta=\theta_{S}(\omega)},
$$


where $\theta_{s}(\omega)$ must satisfy (3.23). Similarly, the power radiated into the solid angle $d \Omega$ is given by $d^{2} P_{s} / d \Omega=\left(e^{2} / 2 \pi c\right)\left\{\omega^{2} n \kappa^{2}\left[-\beta_{1} J_{s}^{\prime}(X)+\left(\alpha_{y} s \beta_{1} / X+\alpha_{z} \beta_{2}\right) J_{s}(X)\right]^{2}\right.$

$$
\left.\cdot[1+(\omega / n) \partial n / \partial \omega] /\left|1-\beta_{2} n \cos \theta[1+(\omega / n) \partial n / \partial \omega]\right|\right\}_{\omega=\omega_{s}(\theta)},
$$

where $\omega_{s}(\theta)$ must satisfy (3.23). (These expressions for the power differ from those given by Eidman [1958] in the factors $\rho, \kappa$, and the sign on $J_{s}^{\prime}$, as well as including relativistic energies.)

Two limiting cases of these power expressions are of special interest. First, consider $\beta_{1}=0$ which gives

$$
d P_{0} / d f=\left(2 \pi e^{2} / c \beta_{2}\right) \omega\left\{\kappa^{2} \alpha_{z}^{2} \beta_{2}^{2} \cdot[1+(\omega / n) \partial n / \partial \omega] /|1-(\partial n / \partial \theta) / n \tan \theta|\right\}_{\theta=\theta_{0}(\omega)}
$$

for the Cerenkov radiation and the cyclotron terms vanish. Secondly, consider $\beta_{2}=0$, which gives $p_{s}=s \omega_{B} / \gamma$ and

$$
d^{2} P_{s} / d \Omega=\left(e^{2} / 2 \pi c\right)\left\{\omega^{2} n \kappa^{2}\left[-\beta_{1} J_{s}^{\prime}(X)+\alpha_{y} s \beta_{1} J_{s}(X) / X\right]^{2} \cdot[1+(\omega / n) \partial n / \partial \omega]\right\}_{\omega=s \omega_{B} / \gamma}
$$

for $s \geqslant 1$. Here only the normal cyclotron emission is present since the motion needed for the anomalous effect is absent. (Although the index is omitted from the emission equation in this case, the frequencies are restricted to the propagation bands of the modes, of course.)

In free space where $n=1, \alpha_{\theta}= \pm 1, \alpha_{k}=0$, the cyclotron terms in (3.29) reduce to the Schott formula [see Landau and Lifshitz, 9-8, 1951] when the power from both modes is added $(s>0$ only since $n=1$ ),

$$
d^{2} P_{s} / d \Omega=\left(e^{2} / 2 \pi c\right)\left(s^{2} \omega_{B}^{2} / \gamma^{2}\right)\left[\beta_{1}^{2} J_{s}^{\prime 2}\left(\beta_{1} s \sin \theta\right)+\cot ^{2} \theta J_{s}^{2}\left(\beta_{1} s \sin \theta\right)\right]
$$

where $X$ from (3.15), $\kappa$ from (3.6), and $\omega=s \omega_{B} / \gamma$ have been introduced explicitly. Since $n \gtrsim 1$, $\alpha_{\theta} \simeq 1$, and $\alpha_{k} \gg 1$ (for $\sin \theta \gg 0$ ) in a magnetoplasma, a comparison of (3.30) and (3.29) shows that the magnitude of the power radiated into a plasma is far greater due to the interaction with the medium, but the emission equation limits the angles of emission (or frequency bandwidth).

Although the power expression $d^{2} P_{s} / d \Omega$ is evaluated at $\theta$ and $\phi(\phi$ is arbitrary by azimuthal symmetry), this power follows a ray path along $\theta^{\prime}$ and $\phi$ where $\theta^{\prime} \neq \theta$, in general. It has been shown by Scott [1950] that the ray path is in the direction of the time-averaged Poynting vector $<\mathbf{S}>=(c / 4 \pi)<\boldsymbol{E}_{e m} \times \boldsymbol{H}_{e m}>$. With the electromagnetic fields defined by (2.22) and a proportional to $\mathbf{E}$ from (2.26), the ray path direction is along

$$
<\mathbf{S}>\alpha<\mathbf{E} \times(\mathbf{k} \times \mathbf{E})>\text {. }
$$

Since $\mathbf{E}$ can have an appreciable component along $\mathbf{k}$ in an anisotropic medium, the electromagnetic energy does not propagate in the $\mathbf{k}$ direction, in general. For the specific $\alpha_{\theta}$ and $\alpha_{k}$ given in the next section, it is readily verified that the direction of $\langle\mathbf{S}\rangle$ is within the acute angle between $\mathbf{k}$ and $\pm \mathbf{B}_{0}$.

\section{Emission in a Cold, Collisionless, Electron Plasma}

In order to evaluate the allowed frequencies and radiated power from a relativistic electron, it is necessary to choose a model for the plasma. In this section the plasma is assumed to be so cold that thermal motion can be neglected, so tenuous that collisions can be neglected, and the frequencies of interest are sufficiently high that ionic motion can be neglected. Such a model is adequate for many physical systems including several aspects of VLF propagation in the magnetosphere. In the present application, however, this elementary model is found to be unsatisfactory at certain frequencies and angles of emission. 
Due to the complexity of the formulas and the large number of independent parameters, the behavior of the emission is demonstrated by several examples. In particular, the specific parameter values are appropriate for trapped-electron orbits at sample points in the geomagnetic field. A brief summary of orbit and medium characteristics for the magnetosphere is given in the appendix.

Since the propagation frequency is an independent variable here, it is convenient to introduce the dimensionless quantities $F$ and $P$ which are defined to be the propagation-cyclotron frequency ratio and the plasma-cyclotron frequency ratio, respectively,

$$
F=\omega / \omega_{B}, \quad P=\omega_{P} / \omega_{B}
$$

The plasma frequency is related to the electron density $N_{0}$ by $\omega_{P}^{2}=4 \pi N_{0} e^{2} / m$. In terms of the spatial configuration in figure 2 and these new variables, the dielectric tensor components for the medium described above have the form [Ginzburg, 1961]

$$
\begin{aligned}
& \epsilon_{x x}=1+P^{2} /\left(1-F^{2}\right), \\
& \epsilon_{\theta \theta}=1-P^{2}\left(\sin ^{2} \theta-F^{2}\right) / F^{2}\left(1-F^{2}\right), \\
& \epsilon_{k k}=1-P^{2}\left(\cos ^{2} \theta-F^{2}\right) / F^{2}\left(1-F^{2}\right), \\
& \epsilon_{x \theta}=-\epsilon_{\theta x}=+i P^{2} \cos \theta / F\left(1-F^{2}\right), \\
& \epsilon_{x k}=-\epsilon_{k x}=+i P^{2} \sin \theta / F\left(1-F^{2}\right), \\
& \epsilon_{\theta k}=+\epsilon_{k \theta}=+P^{2} \cos \theta \sin \theta / F^{2}\left(1-F^{2}\right) .
\end{aligned}
$$

For these specific components, the indices of refraction for the medium are found to be

$$
n_{ \pm}^{2}=1+2 P^{2}\left(P^{2}-F^{2}\right) / D_{ \pm},
$$

with

$$
D_{ \pm}= \pm\left[F^{4} \sin ^{4} \theta+4 F^{2}\left(P^{2}-F^{2}\right)^{2} \cos ^{2} \theta\right]^{1 / 2}-2 F^{2}\left(P^{2}-F^{2}\right)-F^{2} \sin ^{2} \theta
$$

and the polarization coefficients defined in (3.5) are given by

$$
\begin{aligned}
\alpha_{\theta \pm} & =-F \cos \theta /\left[F^{2}+P^{2} /\left(n_{ \pm}^{2}-1\right)\right] \\
& =-2 F\left(P^{2}-F^{2}\right) \cos \theta /\left[-F^{2} \sin ^{2} \theta \pm\left(F^{4} \sin ^{4} \theta+4 F^{2}\left(P^{2}-F^{2}\right)^{2} \cos ^{2} \theta\right)^{1 / 2}\right], \\
\alpha_{k \pm} & =-\left(n_{ \pm}^{2}-1\right) F \sin \theta /\left(P^{2}-F^{2}\right) \\
& =-\left[P^{2} F \sin \theta-\alpha_{\theta \pm} P^{2} \cos \theta \sin \theta\right] /\left[P^{2}\left(\cos ^{2} \theta-F^{2}\right)-F^{2}\left(1-F^{2}\right)\right] .
\end{aligned}
$$

In this notation the upper (+) sign denotes the ordinary (whistler) mode and the lower $(-)$ sign denotes the extraordinary mode of polarization (Ginzburg's nomenclature). The expressions for $\alpha_{\theta \pm}$ and $\alpha_{k \pm}$ describe a wave of the form $\exp i(\omega t-\mathbf{k} \cdot \mathbf{r})$.

The indices given by (4.3) and (4.4) have several unusual properties. They have resonances $\left(n_{ \pm}^{2}= \pm \infty\right)$ at

$$
F_{R \pm}^{2}=\frac{1}{2}\left(P^{2}+1\right) \mp \frac{1}{2}\left[\left(P^{2}+1\right)^{2}-4 P^{2} \cos ^{2} \theta\right]^{1 / 2}
$$




$$
\cos ^{2} \theta_{R}=F^{2}\left[1+\left(1-F^{2}\right) / P^{2}\right]
$$

as well as at $F=0$, and they have cutoffs at

$$
F_{0}=P \quad\left(n_{+}=0\right), \quad F_{X, Z}= \pm \frac{1}{2}+\left(\frac{1}{4}+P^{2}\right)^{1 / 2} \quad\left(n_{-}=0\right) .
$$

From these relations it can be verified that $F_{R_{+}}<P$ and $|\cos \theta|, F_{X}>F_{R_{-}}>P$ and 1 , and $F_{Z}<P$. In a slowly varying medium, electromagnetic energy is absorbed at a resonance and reflected at a cutoff, in general.

In the frequency band $0<F<F_{R+}$, the ordinary mode index $n_{+}$is real and appreciably greater than unity; for $F_{R+}<F<F_{0}, n_{+}$is imaginary (evanescent); and for $F_{0}<F<\infty, n_{+}$asymptotically approaches unity. In the frequency band $0<F<F_{Z}$, the extraordinary mode index $n_{-}$is imaginary; for $F_{Z}<F<F_{R_{-}}, n_{-}$is real and increasing; for $F_{R_{-}}<F<F_{X}, n_{-}$is again imaginary; and for $F_{X}<F<\infty, n_{-}$asymptotically approaches unity.

At very high frequencies where the index is near unity, the radiation from an electron is described approximately by the free-space formulas derived by Schwinger [1949]. For those frequencies where the index is imaginary, emission of electromagnetic radiation cannot occur. Hence, attention will be confined hereafter to the ordinary-mode frequency band $0<F<F_{R+}$, where $n_{+}>1$, and to the extraordinary-mode frequency band $F_{Z}<F<F_{R-}$, where $n_{-}>0$. (Since ionic motion has been neglected, the frequencies are further restricted here to $F>>m / M, P m / M$, where $M$ is the ion mass.)

The ordinary mode frequency band $0<F<F_{R+}$ is often called the whistler mode because VLF radio whistlers propagate in this mode. The whistler-mode index of refraction is plotted in figure 3 as a function of $F$ with $\theta$ parametric and $P=2$. For a given $F$ (and $P$ ) it is apparent that $|\cos \theta|$ $>\cos \theta_{R}$. This mode is frequently represented by its quasi-longitudinal approximation

$$
n_{+}^{2}=1+P^{2} / F(|\cos \theta|-F) \quad \text { for } F^{2} \sin ^{4} \theta / \cos ^{2} \theta<<\left(P^{2}-F^{2}\right)^{2},
$$

and its resonance value, $|\cos \theta|$, is also indicated in figure 3 . When the solution of the emission equation is near the resonance, the discrepancy between $F_{R+}$ and $|\cos \theta|$ can cause serious errors in the power evaluation if the approximation is used.

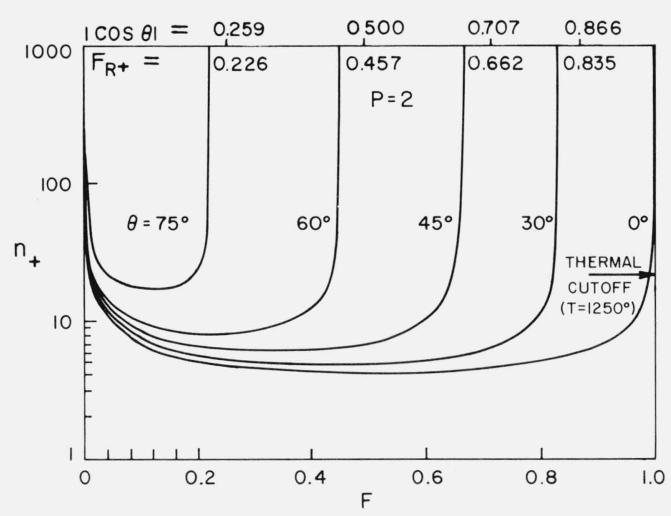

FIGURE 3. The ordinary (whistler) mode index of refraction. The quasi-longitudinal resonance approximation $|\cos \theta|$ and the thermal cutoff for longitudinal $\left(\theta=0^{\circ}\right)$ propagation are also shown. 


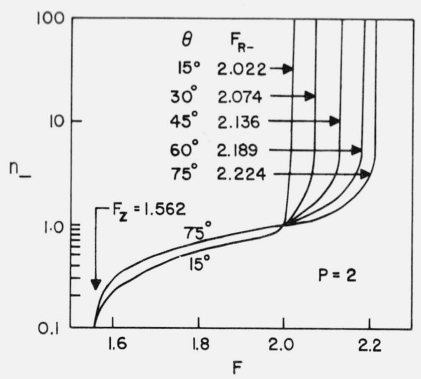

FIGURE 4. The extraordinary mode index of refraction.

The extraordinary-mode frequency band $F_{Z}<F<F_{R-}$ has no special designation nor resonance approximation. It is shown in figure 4 as a function of $F$ with $\theta$ parametric and $P=2$. Here, for a given $F \gtrless P,|\cos \theta| \lessgtr \cos \theta_{R}$.

The polarization of these modes is quite similar. From (4.5) and (4.6) it can be shown that the electric vector of the whistler mode always rotates in the same sense as electrons gyrate about the static field. The rotation in the other mode has the same sense when $F>P$, but is opposite when $F<P$ because $n_{-}<1$.

Although the indices of refraction given in (4.3) are complicated algebraically, when they are substituted into (3.23), the emission equation for both modes of propagation reduces to a quadratic in $\cos ^{2} \theta_{s}(\boldsymbol{F})$,

$$
A \cos ^{4} \theta_{s}+B \cos ^{2} \theta_{s}+C=0
$$

where

$$
\begin{aligned}
& A=\beta_{2}^{4} F^{2}\left[\left(P^{2}-F^{2}\right)^{3}-F^{2}\left(P^{2}-F^{2}\right)\right]+\beta_{2}^{2} P^{2} F^{2}(F-s / \gamma)^{2}, \\
& B=\beta_{2}^{2} F^{2}\left[2\left(P^{2}-F^{2}\right)^{2}+P^{2}-2 F^{2}\right](F-s / \gamma)^{2}-P^{2}(F-s / \gamma)^{4}, \\
& C=F^{2}\left(P^{2}-F^{2}+1\right)(F-s / \gamma)^{4} .
\end{aligned}
$$

The allowable parameter ranges for $\beta_{2}$ and $P$ and allowable frequencies $F$ are determined by the condition that the elementary roots,

$$
\cos ^{2} \theta_{s}^{ \pm}=\left[-B \pm\left(B^{2}-4 A C\right)^{1 / 2}\right] / 2 A,
$$

are real, positive, $\gtrless \cos ^{2} \theta_{R}$ according to the mode, and $\leqslant 1$. These superscripts which denote the two roots should not be confused with the subscripts $( \pm)$ which denote the two modes of propagation. In keeping with the emission directions assigned by (3.23), it is required that $0 \leqslant \theta_{0}^{ \pm} \leqslant \pi / 2$; $0 \leqslant \theta_{s}^{ \pm} \leqslant \pi / 2$ when $F>s / \gamma, s>0$; or $\pi / 2 \leqslant \theta_{s}^{ \pm} \leqslant \pi$ when $F<s / \gamma, s>0$; and $0 \leqslant \theta_{s}^{ \pm} \leqslant \pi / 2$ when $s<0$ irregardless of $F$.

In principle, one could determine $F_{s}(\theta)$, but the equation is an eighth degree polynomial and its analytical properties are more obscure.

Since the two propagation modes are quite different, their emission properties will be discussed separately.

Ordinary- (whistler-) mode emission is discussed first. A typical set of curves for $\cos \theta_{s}^{ \pm}(F)$ are plotted in figure 5 for $s=0, \pm 1, \pm 2, \pm 3$, with $P=2.25$ and several arbitrary values of $\beta_{2}$. The Cerenkov emission is in the forward hemisphere, the normal cyclotron emission is in the backward hemisphere, and the anomalous cyclotron emission is in the forward hemisphere. The $\cos$ $\theta_{s}^{+}$and $\cos \theta_{s}^{-}$regions are adjacent but distinct with a boundary at $\cos \theta_{s}^{\prime}$ defined below. In the 

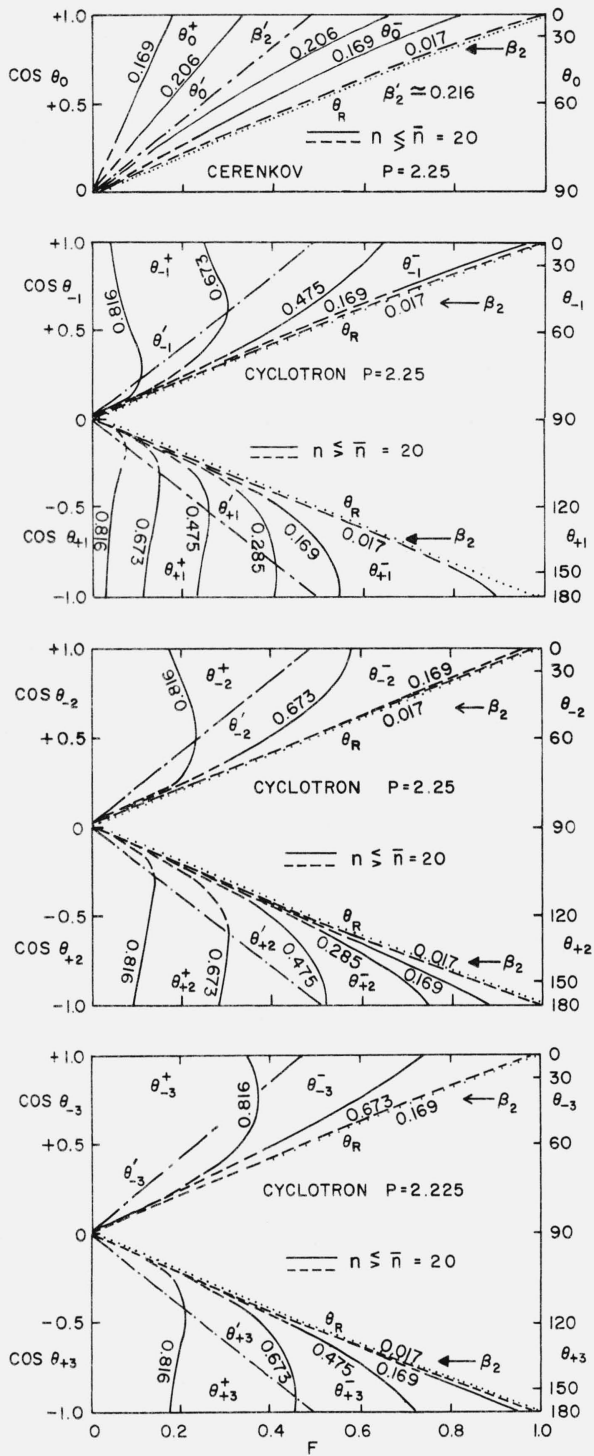

Figure 5. Solutions of the emission equation $\theta_{s}^{ \pm}(\mathrm{F})$ for Cerenkov $(\mathrm{s}=0)$ and normal $(\mathrm{s}>0)$ and anomalous $(\mathrm{s}<0)$ cyclotron radiation in the ordinary (whistler) mode $\left(\beta_{2} / \beta_{1}=\sqrt{3}\right)$.

Cerenkov case, only, there is an upper limit $\beta_{2}^{\prime}$ on $\beta_{2}$ which occurs on the boundary $\cos \theta_{s}^{\prime}$; however, there is always a root for small $\beta_{2}$ when the index is unbounded. It is apparent from the plots that a given frequency may be emitted along two cones defined by $\theta_{s}^{+}$and $\theta_{s}^{-}$, along only one cone defined by $\theta_{s}^{-}$, or may not be allowed at all. An upper bound on the index which is discussed in the next section is also shown.

The solutions of the emission equation for this mode have a few analytical properties which are quite easily demonstrated. In the low frequency limit $F \ll 1\left(P \sim 1, \beta_{2}<1\right)$, the solutions can be expanded in Maclaurin's series,

$\cos ^{2} \theta_{s}^{+}=\left\{(F-s / \gamma)^{2}\left[(F-s / \gamma)^{2}-\beta_{2}^{2}\left(2 P^{2}+1\right) F^{2}\right] / \beta_{2}^{2} F^{2}\left(\beta_{2}^{2} P^{4}+s^{2} / \gamma^{2}\right)\right\}\left[1-0\left(\beta_{2}^{2} F^{2}\right)\right]$,

$$
\cos ^{2} \theta_{s}^{-}=\left\{\cos ^{2} \theta_{R} /\left[1-\beta_{2}^{2}\left(2 P^{2}+1\right) F^{2} /(F-s / \gamma)^{2}\right]\right\}\left[1+0\left(\beta_{2}^{2} F^{2}\right)\right] .
$$


From these expressions it is easily verified that $1>\cos ^{2} \theta_{0}^{+}>\cos ^{2} \theta_{0}^{-}>\cos ^{2} \theta_{R}, \cos ^{2} \theta_{s}^{+}>1$ for $s \neq 0$, and $\cos ^{2} \theta_{s}^{-}$approaches $\cos ^{2} \theta_{R}$ for $s \neq 0$. Hence, as $F$ decreases, $F_{R+}$ decreases faster, so that $n_{+}$approaches the resonance limit at $F_{R+}$ and not the limit at $F=0$. Also, the emission is transverse to $\mathbf{B}_{0}$ when $F \ll 1$. In the Cerenkov limit $\theta=0(s=0)$, the emission equation has the simple solution

$$
F_{0}^{ \pm}(0)=\frac{1}{2} \pm \frac{1}{2}\left(1-4 \beta_{2}^{2} P^{2} \gamma^{2}\right)^{1 / 2} .
$$

Since $F$ must be real, the parameters must satisfy the condition $\beta_{2} P \gamma \leqslant 1 / 2$. In the cyclotron limit $\theta=0$ or $\pi$, the emission equation reduces to an elementary cubic in $F$ which always has one root $F_{s}(0$ or $\pi)$ in the range $0<F<1$. By definition, the boundary between the two solutions is

$$
\cos ^{2} \theta_{s}^{+}=\cos ^{2} \theta_{s}^{-}=-B / 2 A=\cos ^{2} \theta_{s}^{\prime},
$$

and the vanishing discriminant $B^{2}=4 A C$ determines $F_{s}^{\prime}$ and the maximum $\beta_{2}^{\prime}$ for the case $s=0$.

The frequency spectra and the angular patterns of the power given by (3.26) and (3.27) have been evaluated for a few specific parameters. The results for Cerenkov radiation and the first few cyclotron harmonics are presented in figures 6 and 7 . The parameters of the background medium have been assigned the values $f_{B}=\omega_{B} / 2 \pi=102 \mathrm{kc} / \mathrm{s}$ and $f_{P}=\omega_{P} / 2 \pi=229 \mathrm{kc} / \mathrm{s}$, which correspond to the magnetospheric locus $L=3, \lambda=30^{\circ}$ defined in the appendix. The pitch angle $\psi$ between the electron's velocity and $\mathbf{B}_{0}$ has been set at $30^{\circ}$ so that $\beta_{2} / \beta_{1}=\sqrt{3}$. In figures 6 and 7 , the radiated power is shown for the electron kinetic energies $K E=10,1000 \mathrm{keV}$ (dashed and solid curves); the very fine structure involving $\Delta F<0.01$ is omitted. When both roots of the emission equation contribute to the power, the superscripts \pm are explicitly given with the values of $s$. The dotted curves are mean values of the power in regions with very large amplitude fluctuations where the index is unrealistically large. The effects of a bound on the index, which are discussed in the next section, are also included (solid curves only).

These figures demonstrate several unanticipated features of the emission process. First, there is a broad maximum in the Cerenkov spectrum, and the maxima in the cyclotron spectra are not at $F_{1}(\pi)$ as linear emission theories tacitly assume. Second, the angular patterns show that the major portion of the energy is radiated at large emission angles from the static field $\mathbf{B}_{0}$ (wave normal direction, not ray path direction). Third, the energy in the cyclotron harmonics increases with harmonic number. Fourth, the energy radiated by the cyclotron harmonics becomes infinite as $F \rightarrow 0$ or $\theta \rightarrow \pi / 2$. Finally, the power radiated by a nonrelativistic electron increases as $\beta_{2}$ (or $K E$ ) decreases. (The infinite amount of energy indicated by these last three properties is suppressed by introducing thermal, ion, or collisional effects.)

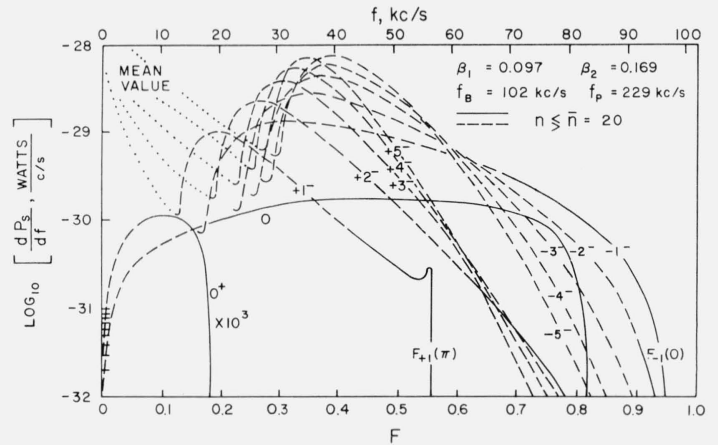

Figure 6a. Frequency spectra of Cerenkov $(\mathrm{s}=0)$ and normal $(\mathrm{s}>0)$ and anomalous $(\mathrm{s}<0)$ cyclotron radiation in the ordinary (whistler) mode from a $10 \mathrm{keV}$ electron in a magnetoplasma.

$$
\left(L=3.0, \lambda=30^{\circ}\right)
$$

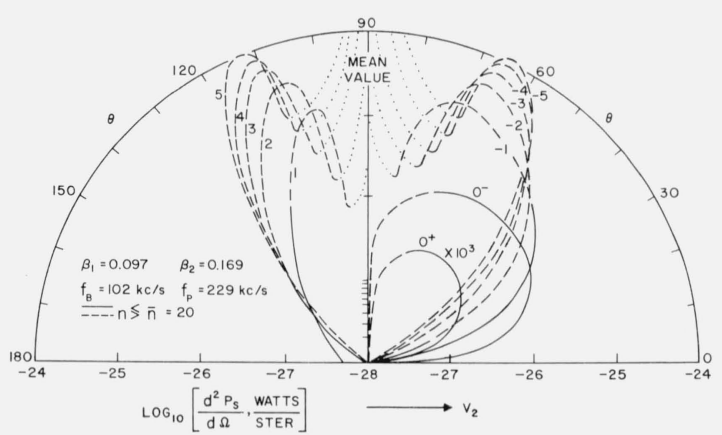

Figure 6b. Angular patterns of Cerenkov $(\mathrm{s}=0)$ and normal $(\mathrm{s}>0)$ and anomalous $(\mathrm{s}<0)$ cyclotron radiation in the ordinary (whistler) mode from a $10 \mathrm{keV}$ electron in a magnetoplasma.

$\left(L=3.0, \lambda=30^{\circ}\right)$ 


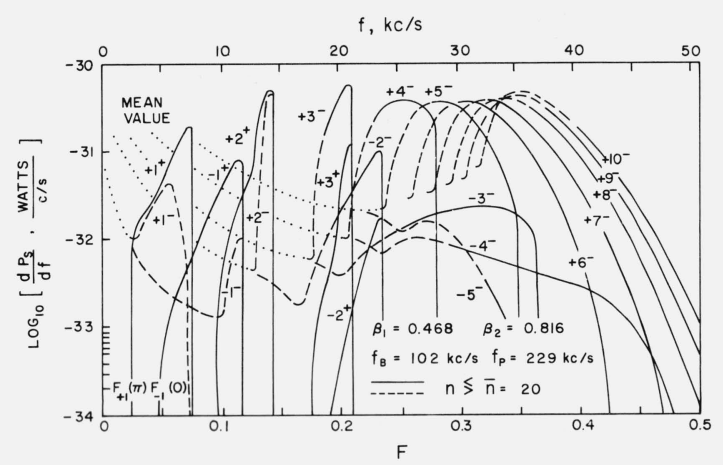

FIGURE 7a. Frequency spectra of normal (s $>0$ ) and anomalous $(\mathrm{s}<0)$ cyclotron radiation in the ordinary (whistler) mode from a $1000 \mathrm{keV}$ electron in a magnetoplasma.

$\left(L=3.0, \lambda=30^{\circ}\right)$

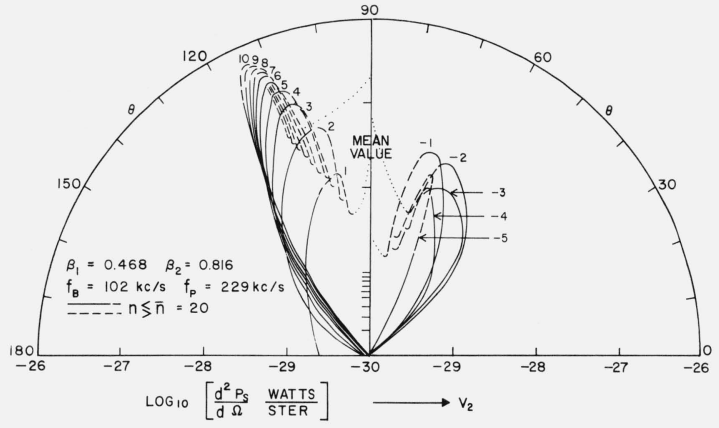

Figure 7b. Angular patterns of normal $(\mathrm{s}>0)$ and anomalous $(\mathrm{s}<0)$ cyclotron radiation in the ordinary (whistler) mode from a $1000 \mathrm{keV}$ electron in a magnetoplasma.

$\left(L=3.0, \lambda=30^{\circ}\right)$

These properties of the power expressions can be demonstrated approximately without performing extensive numerical analysis. From (4.5) and (4.6) it is easily verified that $\left|\alpha_{k}\right| \gg\left|\alpha_{\theta}\right| \simeq 1$ for $0 \ll \theta \ll \pi$. Using this result and the emission equation as a definition of $n$, and assuming that $\left|J_{s}^{\prime}\right| \ll\left|\alpha_{k} J_{s}\right|$ and that the partial derivatives of $n$ are slowly varying, the power expressions are proportional to

$$
\begin{aligned}
& d P_{0} / d f, d^{2} P_{0} / d \Omega \sim F^{3} J_{0}^{2} \tan ^{2} \theta / \beta_{2}^{3}\left(P^{2}-F^{2}\right)^{2} \\
& d P_{s} / d f, d^{2} P_{s} / d \Omega \sim s^{2} F J_{s}^{2} \tan ^{2} \theta / \gamma^{2} \beta_{2}^{3}\left(P^{2}-F^{2}\right)^{2} \quad s \neq 0,
\end{aligned}
$$

for $F \neq P$. For small $F$ the approximation $\cos \theta^{-} \simeq F$ verifies additional frequency properties. When $\theta=0$ or $\pi$, it is found that $\alpha_{k}=0, \alpha_{\theta}=\mp 1, X=0$, and the power expressions vanish for all $s$ except

$$
\begin{aligned}
d P_{1} / d f & =\left[\left(\pi e^{2} / c\right)\left(\omega \beta_{1}^{2} / \beta_{2}\right)\left(1+C_{F}\right) /\left|1-C_{\theta}\right|\right]_{F=F_{1}(\pi),} \\
d^{2} P_{1} / d \Omega & =\left[\left(e^{2} / 4 \pi c\right)\left(\omega^{2} n \beta_{1}^{2}\right)\left(1+C_{F}\right) /\left|1+\beta_{2} n\left(1+C_{F}\right)\right|\right]_{F=F_{1}(\pi),}
\end{aligned}
$$

where

$$
\begin{aligned}
& C_{F}=P^{2}(2 F-1) / 2(1-F)\left(P^{2}-F^{2}+F\right), \\
& C_{\theta}=P^{2} / 2(1-F)\left(P^{2}-F^{2}\right) .
\end{aligned}
$$

In addition to the results at $L=3, \lambda=30^{\circ}, \psi=30^{\circ}$, the power expressions have also been evaluated at $3,15^{\circ}, 19^{\circ} ; 6,45^{\circ}, 19^{\circ}$; and $6,15^{\circ}, 6^{\circ}$, in order to ascertain the effect of varying $f_{B}, f_{P}$, and $\psi$. Although the relevant frequencies vary considerably, the general shape of the frequency spectra and angular patterns do not change appreciably. As a quantitative example, it is found that a $10 \mathrm{keV}$ electron at $6,15^{\circ}, 6^{\circ}$ radiates less than $10^{-33} \mathrm{~W} /(\mathrm{c} / \mathrm{s})$ at frequencies above $1 \mathrm{kc} / \mathrm{s}$ (the lowest considered) although $f_{B}=5.5 \mathrm{kc} / \mathrm{s}$ and $f_{P}=70 \mathrm{kc} / \mathrm{s}$ so that $n$ is large, and furthermore, below $1 \mathrm{kc} / \mathrm{s}$ the cyclotron emission is confined to $90^{\circ}<\theta<100^{\circ}$.

Extraordinary-mode emission is considerably more complicated than ordinary mode emission. 

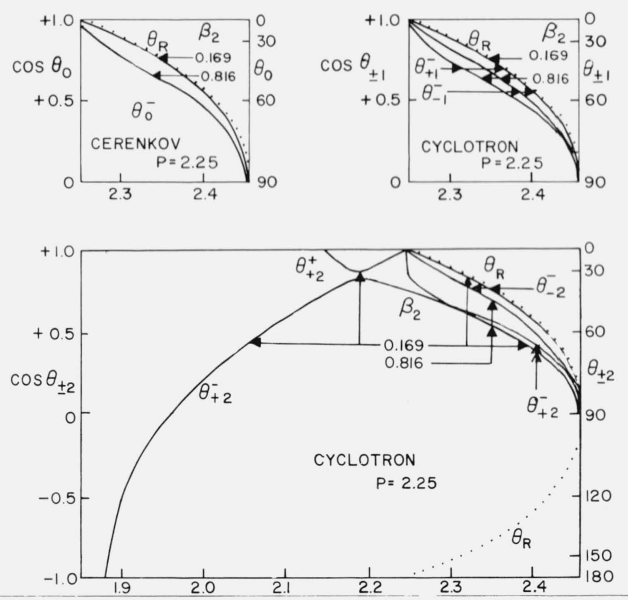

FIGURE 8. Solutions of the emission equation $\theta_{s}^{ \pm}(\mathbf{F})$ for Cerenkov $(\mathrm{s}=0)$ and normal $(\mathrm{s}>0)$ and anomalous $(\mathrm{s}<0)$ cyclotron radiation in the extraordinary mode $\left(\beta_{2} / \beta_{1}=\sqrt{3}\right)$.
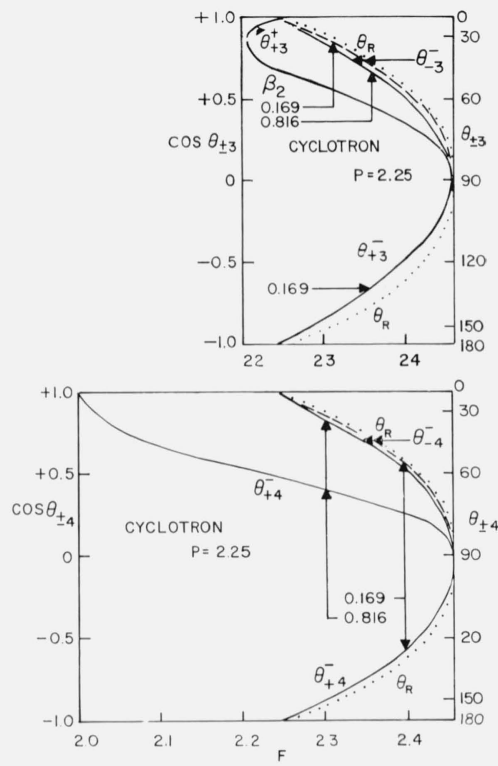

The emission equation roots $\cos \theta_{s}^{ \pm}(F)$ are plotted in figure 8 for $s=0, \pm 1, \pm 2, \pm 3, \pm 4$, with $P=2.25$ and a few arbitrary values of $\beta_{2}$. The Cerenkov emission is again in the forward hemisphere, but the cyclotron emission is spread over both the forward and backward directions. The complicated nature of the solution for $F \sim s / \gamma \leqslant P$ is qualitatively expected from (3.23), and the discontinuity at $F=P$ for small $\beta_{2}$ is attributable to the sharp change in $n_{-}$there.

Emission in this mode is restricted by the index to the frequency band $F_{Z}<F<F_{R-}^{\operatorname{Max}}$ $=\left(P^{2}+1\right)^{1 / 2}$. Some analytical properties of the solutions are observed by expanding them in the limit $\beta_{2}<<1$,

$$
\begin{aligned}
& \cos ^{2} \theta_{s}^{+}=(1-s / \gamma F)^{2} / \beta_{2}^{2}+\delta+\left(P^{2}-F^{2}\right) / P^{2}-\left(P^{2}-F^{2}\right)^{3} / P^{2} F^{2}+0\left(\beta_{2}^{2}\right), \\
& \cos ^{2} \theta_{s}^{-}=\cos ^{2} \theta_{R}\left[1-\beta_{2}^{2} \delta /(1-s / \gamma F)^{2}+0\left(\beta_{2}^{4}\right)\right],
\end{aligned}
$$


where

$$
\delta=2 F^{2} / P^{2}-1-2\left(P^{2}-F^{2}\right)^{2} / P^{2}-\cos ^{2} \theta_{R} .
$$

In general, $F \neq s / \gamma$ so that $\cos ^{2} \theta_{s}^{+}>1$ due to the first term in its expansion and $\cos ^{2} \theta_{s}^{-} \simeq \cos ^{2}$ $\theta_{R}(<1$ for $F>P)$. When $F \simeq s / \gamma$, the root $\cos ^{2} \theta_{s}^{+}$may contribute to the emission pattern depending on the other parameters. However, the expansion for $\cos ^{2} \theta_{s}^{-}$is not valid when $F \simeq s / \gamma$. At $F=P \neq s / \gamma$, it is found that $\delta=0$ and $\cos ^{2} \theta_{R}=1$ so that $\cos ^{2} \theta_{s}^{-} \simeq 1$. Also, at the frequency band limit $F=\left(P^{2}+1\right)^{1 / 2}, \cos ^{2} \theta_{s}^{-} \sim \cos ^{2} \theta_{R}=0$.

The frequency spectra and angular patterns of the Cerenkov and cyclotron power radiated in the extraordinary mode by 10 and $1000 \mathrm{keV}$ electrons at $L=3, \lambda=30^{\circ}$, with $\psi=30^{\circ}$ are shown in figures 9 and 10. In this mode much of the energy is radiated at small angles to the static field $\mathbf{B}_{0}$ by the cyclotron fundamental. The power singularity at $\theta=\pi / 2, F=\left(P^{2}+1\right)^{1 / 2}$ is suppressed by a bound on the index as before. The Cerenkov emission near $\theta=0, F=P$ is not adequately described by this formalism; it consists of electric waves $\left(E_{x}, E_{\theta}=0, E_{k} \neq 0\right)$ which travel with the source $\left(\beta_{2} n=1\right)$ and the infinite power obtained here is invalid. A detailed treatment of this Cerenkov effect has been given by Gerwin and Guernsey [1963].

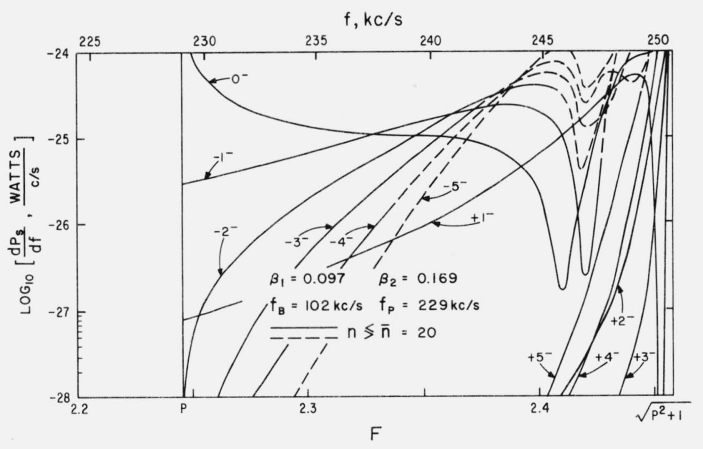

FigURE 9a. Frequency spectra of Cerenkov $(\mathrm{s}=0)$ and normal $(\mathrm{s}>0)$ and anomalous $(\mathrm{s}>0)$ cyclotron radiation in the extraordinary mode from a $10 \mathrm{keV}$ electron in a magnetoplasma.

$$
\left(L=3.0, \lambda=30^{\circ}\right)
$$

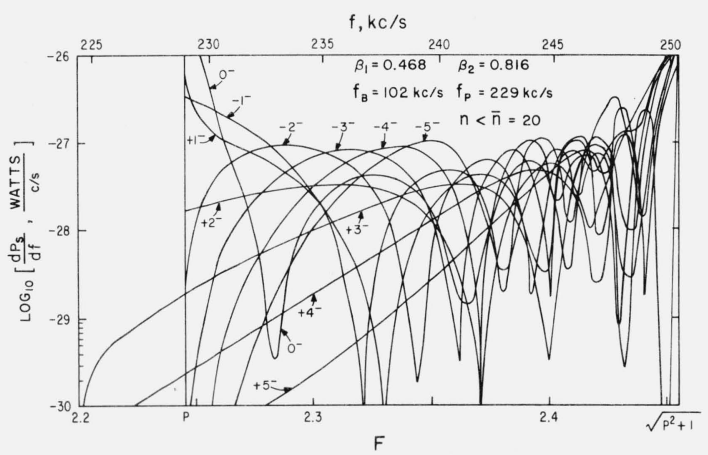

Figure 10a. Frequency spectra of Cerenkov $(\mathrm{s}=0)$ and normal $(\mathrm{s}>0)$ and anomalous $(\mathrm{s}<0)$ cyclotron radiation in the extraordinary mode from a $1000 \mathrm{keV}$ electron in a magnetoplasma.

$$
\left(L=3.0, \lambda=30^{\circ}\right)
$$

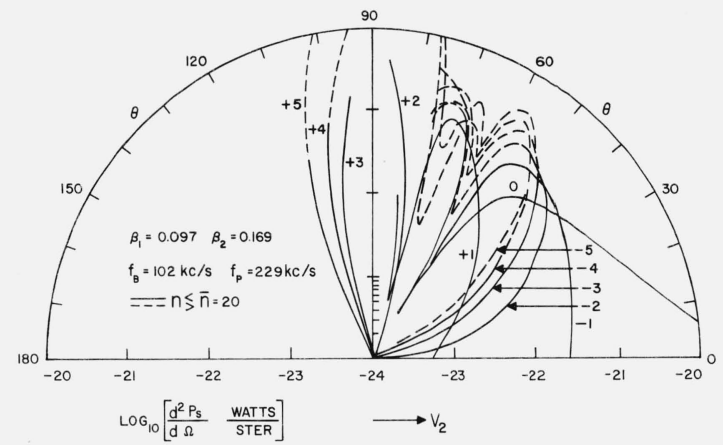

Figure 9b. Angular patterns of Cerenkov ( $\mathrm{s}=0)$ and normal $(\mathrm{s}>0)$ and anomalous $(\mathrm{s}<0)$ cyclotron radiation in the extraordinary mode from a $10 \mathrm{keV}$ electron in a magnetoplasma. $\left(L=3.0, \lambda=30^{\circ}\right)$

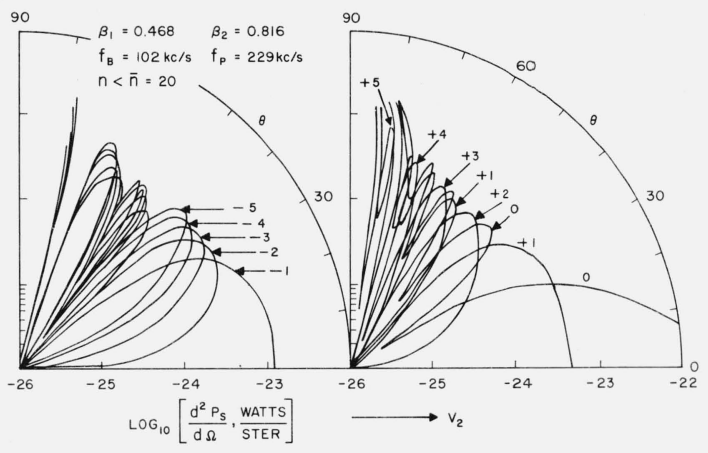

Figure 10b. Angular patterns of Cerenkov $(\mathrm{s}=0)$ and normal $(\mathrm{s}>0)$ and anomalous $(\mathrm{s}<0)$ cyclotron radiation in the extraordinary mode from a $1000 \mathrm{keV}$ electron in a magnetoplasma. 


\section{Discussion}

Since the elementary model plasma described in the last section leads to the emission of an infinite amount of energy by the gyrating electron, it is necessary to introduce some additional effects which will limit the emission and give a physically meaningful result. The major factors which were omitted previously are thermal motion, ionic motion, and collisions in the background medium. For VLF propagation in the magnetosphere and certain other applications, the dominant modification of the propagation properties is obtained by including thermal motion alone.

Mathematically, the boundless energy radiated into the plasma medium is due to the unbounded property of $n_{ \pm}$at $F_{R_{ \pm}}$. From figures 5 and 8 it is evident that the solution of the emission equation approaches $\theta_{R}$ when $F$ approaches $0, \theta$ approaches $\pi / 2$, or $|s|$ increases, and the power increases sharply because $\alpha_{k}$ is proportional to $n^{2}$. Consequently, a physical process which introduces a finite upper bound $\dot{\bar{n}}_{ \pm}$on the index is essential if the radiation is to have physical meaning. Furthermore, such a bound implies $\left|\cos \theta_{s}^{-}\right| \gtrless\left|\cos \bar{\theta}_{ \pm}\right|, F \gtrless \bar{F}_{ \pm},|s|<\bar{s}_{ \pm}$, and $\beta_{2}>\bar{\beta}_{2_{ \pm}}$ according to (3.23), and the values of these limits depend on the explicit form of $n_{ \pm}$.

An artificial bound on the index is introduced here to demonstrate the qualitative effect that it produces on the frequency spectra and angular patterns of the radiated power. The index is assumed to be given by (4.3) when $n_{ \pm}<\bar{n}_{ \pm}$, and by $\bar{n}_{ \pm}$when $n_{ \pm}>\bar{n}_{ \pm}$. Since $n_{ \pm}$increases monotonically as $\left|\cos \theta_{s}^{-}\right|$approaches $\cos \theta_{R}$, the solution of the emission equation is cutoff at the discontinuity where $\left|\cos \theta_{s}^{-}\right|=\left|\cos \bar{\theta}_{ \pm}\right|$. In figures 5 to 10 the cutoff is shown for the case $\bar{n}=20$. The finite power for this artificial case is described by the solid curves. Although the sharp edges would disappear for a continuous index, the dominant maxima are expected to remain. It is noteworthy that the nonrelativistic maxima in both propagation modes occur at the cutoff (figs. 6 and 9) so that a small change in the cutoff location produces a large change in the radiated power. The extent to which this artificial case is quantitatively applicable to a real case depends on the validity of the index approximation which is discussed below.

Insofar as the index approximation is valid, a comparison can be made between the power in the different harmonics and between different electron energies. For this purpose the power expressions were evaluated at a few intermediate energies (not shown). In general, the maximum power in the normal and anomalous cyclotron harmonics, and the Cerenkov radiation (when it exists) is comparable at a given energy. The total radiated power is a slowly decreasing function of $\beta_{2}$ (or kinetic energy for a fixed pitch angle) at nonrelativistic speeds, with an average level of $10^{-30} \mathrm{~W} /(\mathrm{c} / \mathrm{s})$ in the ordinary mode and $10^{-25} \mathrm{~W} /(\mathrm{c} / \mathrm{s})$ in the extraordinary mode. The range of speeds $\beta_{2}$, which allow Cerenkov radiation, is severely restricted from below by a bounded index and above by lack of a polarization interaction. At relativistic speeds the higher cyclotron harmonics dominate, but they are restricted by the upper bound $\bar{s}$.

The formulas for the dielectric tensors, polarization coefficients, and refractive indices which include additional effects are quite complicated and difficult to apply. The extent of their influence on the results is evaluated approximately here by considering some special cases.

Random thermal motion is considered first. Since it tends to disorganize the ordered motion which is essential for wave propagation, there is a minimum phase velocity (or $\bar{n}_{ \pm}$) which the thermal medium can support. The dielectric tensor for a thermal medium is known, and an extensive review of the theory has been given by Stix [1962]. However, the dispersion equation is so complicated, in general, that it has only been solved in a few special cases. In particular for $\theta=0$ (or $\pi$ ), the maximum occurs at $F=1$, and for a Maxwellian distribution with temperature $T$, its value is $\bar{n}_{+} \simeq 3^{1 / 2} P^{2 / 3}\left(\pi \mathrm{Mc}^{2} / K T\right)^{1 / 6} / 2$. Liemohn and Scarf [1963] used a bell-shaped algebraic distribution which gives a much simpler dispersion equation to study the longitudinal whistler mode $n_{+}(\theta=0)$. They found that the zero-temperature index is a valid approximation when $1-F>>\left(K T / \mathrm{Mc}^{2}\right)^{1 / 2} n_{+}$. As yet $n_{+}$has not been studied for arbitrary $\theta$, and $n_{-}$has not been considered at all, although similar behavior is anticipated.

It is impossible to accurately assess the thermal effect on the emission with this limited amount of information about the index. Nevertheless some speculation is conceivable. For the magnetospheric case $T=1250{ }^{\circ} \mathrm{K}$ and $P=2.25$, the zero-temperature index for $\theta=0$ or $\pi$ is valid 
when $1-F>>0.02$ and $n_{+} \leqslant \bar{n}_{+}=20$ (see fig. 3). Hence, the artificial discontinuous index used in figures 5 to 7 is a good approximation when $|\cos \theta|>>0$. At other angles of emission and in the extraordinary mode the validity of the index approximation is unknown at present.

In a thermal magnetoplasma the electromagnetic waves are also absorbed as they propagate so that the power expressions are only valid near the source. The absorption is due to the cyclotron resonance mechanism. The component of thermal motion parallel to $\mathbf{B}_{0}$ causes a Doppler shift of the propagation frequency in the rest frame of each electron. For each angle of propagation there is a corresponding velocity component which shifts the propagation frequency to the resonance. Hence, the damping decrement depends on the frequency and angle of propagation as well as the thermal velocity distribution of the electrons. Its value is determined by the general solution of the dispersion equation. Due to the frequency and angular dependence, the power spectra and patterns will change as the signal propagates away from the source. Fortunately, in many applications the temperature of the plasma is so low that very few electrons resonate and the thermal attenuation is negligible. If the background plasma has an appreciable number of "fast" electrons due to a high temperature or other sources, however, the damping must be considered.

In a recent series of papers, several limiting cases of emission in a thermal magnetoplasma are analyzed by Pakhomov, Stepanov, and Aleksin [1962], Pakhomov and Stepanov [1963a, 1963b]. In general, their analysis is restricted to the index values $n_{ \pm} \sim 1$ where several approximations for the emission equation and the power could be made. When the limitation on $n_{ \pm}$is relaxed, other restrictions on $\omega$ or $\theta$ are imposed. Hence, their results cover a variety of special cases but do not provide information about the complete frequency spectra and emission patterns. A convenient feature of their expressions is that the thermal attenuation factors are included explicitly.

Due to the inertia of the ions, they only interact appreciably with the electromagnetic fields when $F<<1$. In this region Hines [1957] has shown that the index is bounded for $\theta \sim \theta_{R}$ and ionic effects are important when $F<(\mathrm{m} / \mathrm{M})^{1 / 2}$. Since $\cos \theta^{-} \sim F$ when $P>1$ according to (4.14), the limit is also applicable to the angle of emission. For the particular case of ionized hydrogen, the limit is so close to the transverse direction that the bound imposed on the power is extremely large compared to the anticipated thermal bound. Consequently, ionic effects can usually be disregarded.

Collisions which the electrons make with heavy particles in the medium are usually approximated by a viscous damping force in the equation of motion. Such a force restricts the wave propagation in a manner similar to thermal motion. The theory has been reviewed by Ratcliffe [1959], Ginzburg [1961] and others. In general, collisions are only important when the collision frequency $\omega_{c}$ satisfies $\omega_{c} \gtrsim \omega$. In the magnetosphere, $\omega_{c}<10 \mathrm{rad} / \mathrm{sec}$ so that collisions are negligible in the VLF band. Unfortunately, the dielectric tensor for this elementary theory is not hermitian so that when collisions must be included a different formulation of the problem is needed.

\section{VLF and LF Emissions}

The foregoing properties of the radiation from electrons in a magnetoplasma suggest some conclusions about the role that electron emissions might play in the generation of VLF and LF emissions. An important test for any prospective source is to compare the theoretical and observed power of the signal. Also, since the emissions have a wide variety of discrete dispersion patterns as well as continuous bands, some mechanism is needed to provide for frequency selection. Finally, the emission properties suggest another strong signal which is only observable by satellites or rockets.

In the magnetosphere, the geomagnetic field and the plasma density vary so slowly in space and time that the propagation theory for uniform media is usually acceptable. However, the magnetosphere changes significantly over several wavelengths so that a signal which propagates in one region may be absorbed at a resonance or reflected at a cutoff in a nearby region. A further complication is presented by the ionosphere where the electron density increases sharply. On the 
geomagnetic equator the cyclotron frequency varies continuously as the inverse cube of geocentric distance from $\sim 1 \mathrm{Mc} / \mathrm{s}$ at the earth's surface to $\sim 1 \mathrm{kc} / \mathrm{s}$ near 10 earth radii. The variation of the plasma frequency is very similar with a range from $\sim 5 \mathrm{Mc} / \mathrm{s}$ in the $F 2$ peak of the ionosphere to $\sim 20 \mathrm{kc} / \mathrm{s}$ near 10 earth radii. Mathematical models of the region which include latitude effects are presented in the appendix. In the magnetosphere, $P$ varies from $\sim 0.03$ to $\sim 20$.

At the conclusion of section 3 , it was noted that the ray path followed by the electromagnetic energy is not in the wave normal direction $(\theta)$, but rather is in the time-average Poynting vector direction much closer to the magnetic field direction. As a result, it is frequently assumed that the ray path follows a magnetic flux tube. While such an approximation is questionable, it provides a convenient framework for discussing some general propagation properties of electron emissions from their sources to potential receivers.

Due to the cutoffs and resonances of the modes, the signals only propagate in well defined regions of the magnetosphere. In the whistler mode the emitted frequencies can always propagate into regions of increasing $\omega_{B}$ (inward), but can only propagate into regions of decreasing $\omega_{B}$ (outward) if $F<F_{R+}$ (neglecting thermal and collisional effects). In the extraordinary mode the emitted frequencies can propagate into regions of increasing $\omega_{B}$ until $F=F_{Z}$, where they are reflected and can propagate into regions of decreasing $\omega_{B}$ if $F<F_{R-}$. Hence, the signals in the whistler mode can penetrate the ionosphere and reach the ground, whereas signals in the other mode are reflected, in general, above the ionosphere, and both modes have a minimum upper frequency limit which occurs at the equator. A schematic diagram of possible propagation paths is shown in figure 11. Since part of the whistler mode energy is reflected at the ionosphere by irregularities, an emission may echo between hemispheres. Similar echoing in the extraordinary mode is only possible when the source is very near the equator.

A detailed calculation of the VLF and LF power which is radiated into the magnetosphere is impossible at this time because the propagation properties are not fully understood and the phase space distribution of the electrons is unknown to a large extent. Hence, some crude estimates of the power from incoherent and coherent sources must suffice.

First, it will be shown with a specific numerical calculation that incoherent electron emissions apparently cannot account for the observed emissions received on the ground in the whistler mode. The density of magnetospheric electrons $N_{e}$ which emit appreciable radiation is assumed to be $\sim 0.1 \mathrm{elect} / \mathrm{cm}^{3}$, which is fairly consistent with satellite measurements reported by O'Brien [1962] and whistler analysis by Liemohn and Scarf [1964]. The volume of a flux tube with a $1 \mathrm{~cm}^{2}$ base which crosses the equator at 3 earth radii $(L=3)$ is found to be $\sim 10^{10} \mathrm{~cm}^{3}$. On the basis of the numerical results for the ordinary mode, the average emitted power $P_{e}$ along the flux tube is assumed to be on the order of $\sim 10^{-30} \mathrm{~W} /(\mathrm{c} / \mathrm{s})$. With these gross approximations the incoherent power at the base of the flux tube is found to be $10^{-21} \mathrm{~W} / \mathrm{cm}^{2}(\mathrm{c} / \mathrm{s})$. This is several orders of magnitude below the observed power level $P_{r}$ of $10^{-14} \mathrm{~W} / \mathrm{cm}^{2}(\mathrm{c} / \mathrm{s})$ at the base of the magnetosphere (above the ionosphere).

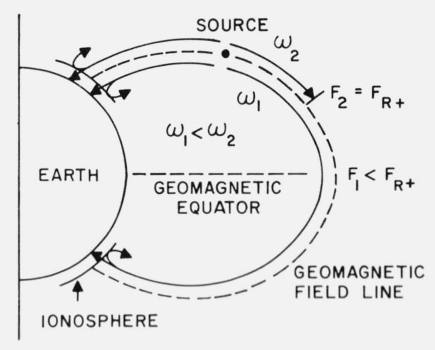

Figure 1la. Possible ray (energy) paths for the ordinary (whistler) mode of propagation in the magnetosphere.

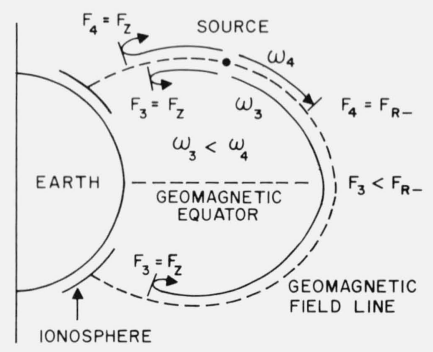

Figure 11b. Possible ray (energy) paths for the extraordinary mode of propagation in the magnetosphere. 
Second, coherent emissions from "bunches" of electrons are shown to be a feasible source of the emissions. Since the electromagnetic fields of individual sources must be in phase for coherent radiation, the total power is proportional to the number of emitters squared. The required size of the bunch is determined by equating the observed power over area $A_{r}$ to the emitted power from an extended source of cross section $A_{e}$ and length $l_{e}$ with coherent efficiency $\epsilon$.

$$
A_{r} P_{r}=P_{e}\left(\epsilon N_{e} A_{e} l_{e}\right)^{2} .
$$

The radiation is assumed to be confined to a flux tube so that $B_{r} A_{r}=B_{e} A_{e}$. Then, with the values of $P_{r}, P_{e}$, and $N_{e}$ given above and the arbitrary assumptions $B_{e} / B_{r}=0.1$ and $\epsilon=0.001$, eq (6.1) reduces to $A_{e} l_{e}^{2}=10^{+23} \mathrm{~cm}^{4}$. Hence, the dimensions of the bunches are small relative to the magnetosphere, and the scale is comparable to a few wavelengths. On the basis of this crude calculation, it appears feasible that coherent emission from electron bunches is a conceivable source of the emissions in the ordinary mode.

Since the electron emissions are continuous over a wide band of frequencies, the discrete nature of the observed VLF emissions must be attributed to the coherent interaction. The triggering of emissions, which Helliwell [1963] has observed, suggests that strong whistler mode signals from lightning strokes interact with the electrons and organize their phase space distribution so that specific frequencies are amplified by coherence. The various interaction mechanisms which may produce this bunching have been reviewed by Brice [1964].

The one-dimensional calculations by Dowden [1962c] and others, which support the theory that emission at the Doppler-shifted fundamental cyclotron frequency $\left(F_{1}(\pi)\right)$ is responsible for hooks, is not ruled out by these results, but it is much more likely that stronger emissions at other frequencies in nonlongitudinal directions dominate the coherent signals. The traveling wave tube amplification of incoherent signals which is proposed by Gallet and Helliwell [1959] seems improbable because a gain of several orders of magnitude is required.

The power in the extraordinary mode is considerably stronger than that in the ordinary mode above the ionosphere. Hence, much less coherence is necessary for this mode to produce signals which are detectable in the presence of the observed whistler mode noise level. The average power level is on the order of $10^{-25} \mathrm{~W} /(\mathrm{c} / \mathrm{s})$ so that $10^{9}$ electrons in the flux tube described above produce $10^{-16} \mathrm{~W} / \mathrm{cm}^{2}(\mathrm{c} / \mathrm{s})$ of incoherent power at the base of the tube. Rocket observations by Walsh, Haddock, and Schulte [1963] have revealed intense noise signals in the extraordinary mode so that this mode cannot be ignored.

Certainly the calculations presented here are based on very limited numerical results and must be considered as only preliminary estimates of the power levels. A thorough quantitative analysis must await the inclusion of additional limiting physical effects such as thermal motion and perhaps collisions. However, they serve to point out some inherent weaknesses in the existing theories for VLF emissions in the ordinary mode, and they point out complications by the extraordinary mode when observations are made above the ionosphere. Furthermore, order of magnitude calculations of the power provide an additional test for future theories of VLF and LF emissions which are based on charged particle radiation in a plasma.

I thank J. A. Fejer, W. B. Hanson, and J. E. Midgley for many discussions and helpful suggestions during the preparation of this paper. The assistance of L. B. Wadel, who programmed the computations, is gratefully acknowledged. This research has been supported by the National Aeronautics and Space Administration under grant NsG-269-62. 


\section{Appendix}

The magnetic field of the earth is closely approximated by a dipole field of the form

$$
\mathbf{B}_{0}=b(-2 \sin \lambda \hat{R}+\cos \lambda \hat{\lambda}) / R^{3},
$$

where $R$ is the geocentric radial distance and $\lambda$ is geomagnetic latitude. With $R$ expressed in earth radii the dipole moment $b$ is 0.313 gauss (earth radii) ${ }^{3}$. The field line equation is

$$
R=L \cos ^{2} \lambda,
$$

where $L$ is the radial distance at which the field line intercepts the equator (McIlwain $L$ parameter). The scalar field is given by

$$
B_{0}=b\left(1+3 \sin ^{2} \lambda\right)^{1 / 2} / L^{3} \cos ^{6} \lambda .
$$

Several mathematical models which closely approximate the thermal plasma density in the magnetosphere have been proposed. In this analysis the field-line diffusion model by F. S. Johnson [1962] is used:

$$
N_{0}=12500 R^{-3} \cos ^{6} \lambda \exp \left(G R^{-1} \sin ^{2} \lambda\right) \quad R>1.3
$$

where $G=M g R_{e} / 2 K T$ is the scale factor of the medium in earth radii $\left(R_{e}\right)$. The temperature of the plasma $T$ is usually assumed to be $\sim 1250^{\circ} \mathrm{K}$, which gives $G=3$.

The motion of high-energy electrons in this medium is described by the first-order orbit theory for trapped particles [Welch and Whitaker, 1959; Chamberlain, 1963]. The electrons are constrained to follow helical orbits along magnetic flux tubes and are reflected at conjugate mirror points in opposite hemispheres. The longitudinal motion is neglected here. Due to conservation of the electron's magnetic moment, the pitch angle $\psi$ between the velocity and field vectors is related to its equatorial value $\psi_{e}$ by

$$
\sin \psi=\left(1+3 \sin ^{2} \lambda\right)^{1 / 4} \sin \psi_{e} / \cos ^{3} \lambda .
$$

The latitude of the mirror point $\lambda_{m}$ is determined by setting $\psi=\pi / 2$ in this equation. Hence, the orbit is completely specified by only two parameters, $L$ and $\psi_{e}$, which are independent of the kinetic energy.

\section{References}

Barrington, R. E., and J. S. Belrose (1963), Preliminary results from the VLF receiver aboard Canada's Alouette satellite, Goddard Space Flight Center Publication X-615-63-36.

Bazhanova, A. E., and V. D. Shafranov (1963), Radiation from a charge moving in plasma close to cyclotron resonance, Dokl. Akad. Nauk SSSR 149, 1049-1051, or Soviet Phys. Doklady 8, 356-358.

Bell, T. F., and O. Buneman (1964), Plasma instability in the whistler mode caused by a gyrating electron stream, Phys. Rev. 133, A1300-A1302.

Brice, N. (1964), Fundamentals of VLF emission generation mechanisms, J. Geophys. Res. 69, 4515-4522.

Chamberlain, J. W. (1963), Motion of charged particles in the earth's magnetic field, in Geophys. eds. C. DeWitt, J. Hieblot, and A. LeBeau, 143-174 (Gordon and Breach, New York, N.Y.).

Dowden, R. L. (1962a), Wide-band bursts of VLF radio noise (hiss) at Hobart, Aust. J. Phys. 15, 114-119.

Dowden, R. L. (1962b), Theory of generation of exospheric very-low-frequency noise (hiss), J. Geophys. Res. 67, $2223-2230$.

Dowden, R. L. (1962c), Doppler-shifted cyclotron radiation from electrons: a theory of very-low-frequency emissions from the exosphere, J. Geophys. Res. 67, 1745-1750. 
Dowden, R. L. (1963), Doppler shifted cyclotron generation of exospheric very-low-frequency noise (hiss), Planetary Space Sci. 11, 361-369.

Eidman, V. Ia. (1958), The radiation from an electron moving in a magnetoactive plasma, J. Exptl. Theoret. Phys. (U.S.S.R.) 34, 131-138, or Soviet Phys. JETP 7, 91-95; Corrections, J. Exptl. Theoret. Phys. (U.S.S.R.) 36, 1335-1336 or Soviet Phys. JETP 9, 947.

Ellis, G. R. A. (1962), Cyclotron radiation from Jupiter, Aust. J. Phys. 15, 344-353.

Franck, I. M. (1942), Bulletin (Izv.) Acad. Sci. U.S.S.R. Phys. Ser. 6, 3.

Gallet, R. M. (1959), The very-low-frequency emissions generated in the earth's exosphere, Proc. IRE 47, $211-231$.

Gallet, R. M. and R. A. Helliwell (1959), Origin of very-low-frequency emissions, J. Res. NBS 63D (Radio Prop.), No. 1, 21-27.

Gerwin, R. and R. Guernsey (1963), On the plasma Cerenkov radiation, Boeing Document D1-82-0263.

Ginzburg, V. L. (1961), Propagation of Electromagnetic Waves in Plasma, ch. III (Gordon and Breach, New York).

Gurnett, D. A. (1963), Very-low-frequency electromagnetic emissions observed with the ONR/SUI satellite Injun III, M. S. Thesis, State Univ. of Iowa, SUI 63-27, J. Geophys. Res. 69, 65-90.

Hansen, S. F. (1963), A mechanism for the production of certain types of VLF emissions, J. Geophys. Res. 68, 5925-5936.

Heitler, W. (1954), The Quantum Theory of Radiation, ch. 1, 3rd edition (Clarendon Press, Oxford, Eng.).

Helliwell, R. A. (1963), Whistler-triggered periodic very-low-frequency noise (hiss), J. Geophys. Res. 68, 5387-5396.

Hines, C. O. (1957), Heavy-ion effects in audio-frequency radio propagation, J. Atmospheric Terrest. Phys. 11, 36-42.

Johnson, F. S. (1962), Physics of the distribution of ionized particles in the exosphere, Electron Density Profiles, ed. B. Maehlum, 404-413, (MacMillan, New York).

Johnson, P. S. (1962), Cerenkov radiation for a cold magnetoactive plasma, Phys. Fluids 5, 118-120.

Kolomenski, A. A. (1953), On electrodynamic gyrotropic media, J. Exptl. Theoret. Phys. (U.S.S.R.) 24, 167-176.

Landau, L. and Lifshitz (1951), The classical theory of fields (Addison-Wesley, Reading, Mass.).

Liemohn, H. B. and F. L. Scarf (1963), Dispersion function for a plasma with a Cauchy equilibrium distribution, Phys. Fluids 6, 388-393.

Liemohn, H. B. and F. L. Scarf (1964), Whistler determination of electron energy and density distributions in the magnetosphere, J. Geophys. Res. 69, 883-904.

MacArthur, J. W. (1959), Theory of the origin of the very low-frequency radio emissions from the earth's exosphere, Phys. Rev. Letters 2, 491-492.

McKenzie, J. F. (1963), Cerenkov radiation in a magneto-ionic medium (with application to the generation of low-frequency electromagnetic radiation in the exosphere by the passage of charged corpuscular streams), Phil. Trans. Roy. Soc. London A255, 585-606.

Murcray, W. B. and J. H. Pope (1960a), Doppler-shifted cyclotron frequency radiation from protons in the exosphere, Phys. Rev. Letters 4, 5-6.

Murcray, W. B. and J. H. Pope (1960b), Radiation from protons of auroral energy in the vicinity of the earth, J. Geophys. Res. 65, 3569-3574.

O'Brien, B. J. (1962), Review of studies of trapped radiation with satellite-borne appratus, Space Science Reviews 1, 415-484.

Pakhomov, V. I., V. F. Aleksin, and K. N. Stepanov (1962), The radiation from an electron moving in a spiral in a magnetoactive plasma I, Soviet Phys. - Tech. Phys. 6, 856-866.

Pakhomov, V. I., and K. N. Stepanov (1963a), The radiation of electrons moving on a spiral in a magnetoactive plasma II, Soviet Phys. - Tech. Phys. 8, 28-33.

Pakhomov, V. I. and K. N. Stepanov (1963b), Radiation of an electron moving in a helical path in a magnetoactive plasma III, Soviet Phys. - Tech. Phys. 8, 325-329.

Ratcliffe, J. A. (1959), The Magneto-ionic theory and its applications to the ionosphere (Cambridge University press, Cambridge, London).

Schiff, L. I. (1955), Quantum Mechanics, sec. 11, 2d edition (McGraw-Hill, New York, N.Y.).

Schwinger, J. (1949), On the classical radiation of accelerated electrons, Phys. Rev. 75, 1912-1925.

Scott, J. C. W. (1950), The Poynting vector in the ionosphere, Proc. IRE. 38, 1057-1068.

Stix, T. H. (1962), The Theory of Plasma Waves (McGraw-Hill Book Co., Inc., New York, N.Y.).

Walsh, D., F. T. Haddock, and H. F. Schulte (1963), Cosmic radio intensities at 1.225 and $2.0 \mathrm{Mc} / \mathrm{s}$ measured up to an altitude of $1700 \mathrm{~km}$, University of Michigan Report 48104.

Watts, J. M., J. A. Koch, and R. M. Gallet (1963), Observations and results from the "hiss recorder," an instrument to continuously observe the VLF emissions, J. Res. NBS 67D (Radio Prop.) No. 5, 569-579.

Welch, J. A., and W. A. Whitaker (1959), Theory of geomagnetically trapped electrons from an artificial source, J. Geophys. Res. 64, 909-922. 